\title{
STRUTTURE MONASTICHE, GESTIONE E MOMENTI DI VITA QUOTIDIANA NEL REGISTRUM EPISTULARUM DI GREGORIO MAGNO
}

\section{CARMELINA URSO}

\author{
UDC: $27-788$ "04/09" \\ 27-43 \\ Original scientific paper \\ Manuscript received: 14. 10. 2016. \\ Revised manuscript accepted: 27. 01. 2017. \\ DOI: 10.1484/J.HAM.5.113703
}

\author{
C. Urso \\ Università degli Studi di Catania \\ Dipartimento di Scienze della Formazione \\ Via Teatro Greco, 84 - 95124 Catania \\ Italia \\ curso@unict.it
}

The study retrieves in Gregory the Great's Registrum epistularum the measures taken on the monastic building, on the management by monastic leaders and on the moral decay that more and more marked the strict monastic setting of the sixth century. The aim is to find in Gregory's action the aspects and the characters of a conscious "monastic policy", thus overcoming at least in part the disagreement which also has been authoritatively formulated.

Keywords: Early Middle Ages, Gregory the Great, Monasticism, Economic and social context

Il mio contributo si inserisce nelle linee programmatiche individuate dagli organizzatori del Colloquium - per i quali «la vita monastica indagata con gli occhi dei monaci è la prospettiva del XXIII Simposio internazionale IRCLAMA» - avvalendosi dello sguardo di Gregorio Magno, il cui pontificato $(590-604)^{1}$ si distinse indubbiamente come un momento di grande potenziamento per il movimento monastico nell'intero Occidente. Né poteva essere altrimenti se si valutano la sua esperienza personale e la sua inclinazione per la vita regolare. È noto che egli fu "costretto" ad abbandonare il rifugio cenobiale che aveva allestito nella Roma del tempo per raggiungere Costantinopoli quale apocrisario di papa Pelagio II, e che non poche volte espresse il suo rammarico per non avere potuto, una volta rientrato a Roma, restituirsi definitivamente al chiostro. Fu presto chiamato a furor di popolo a ricoprire la carica pontificia. Insomma, forse non è corretto definire Gregorio "papa monaco"2, ma non si può trascurare la valenza del suo trascorso monastico.
Salito al trono di Pietro, non tralasciò di occuparsi e preoccuparsi del fenomeno in tutte le sue sfaccettature: favorì l'impianto di nuovi monasteri, da lui intesi idealmente come luoghi di riposo e di pace 3 , ne sorvegliò la realizzazione e ne stigmatizzò i ritardi; fu pronto a sostenere le comunità che vivevano momenti di estrema indigenza; denunciò e perseguì ogni devianza e ogni comportamento immorale; fu soprattutto pronto a sostituirsi agli ordinari locali, per deliberazione canonica preposti al controllo e alla cura delle fondazioni monastiche diocesane, quando costoro palesavano poca attenzione o poca capacità gestionale ${ }^{4}$. Non sempre le sue indicazioni furono adottate dai vari interlocutori; non sempre le sue ammonizioni sortirono gli effetti sperati: dimostrò però di volere assistere con sollecitudine pastorale ogni pecorella del suo gregge. I valori cui fu ispirata l'intera sua azione, pur basati nello specifico dell'ambito monastico su taluni noti principi della regola benedettina e specie sulla stabilitas loci5 e sulla paupertas, come si evidenzia nei

\footnotetext{
${ }^{1}$ Su Gregorio Magno e sul suo pontificato, mi limito a segnalare tra la produzione scientifica più recente, J. RICHARDS, Consul of God: the life and times of Gregory the Great, London, 1980; V. PARONETTO, Gregorio Magno. Un maestro alle origini cristiane d'Europa, Roma, 1983; R.A. MARKUS, Gregory the Great and his world, Cambridge, 1997; J. MOORHEAD, Gregory the Great, London-New York, 2005; B. MÜLLER, Führung im Denken und Handeln Gregors des Grossen, Tübingen, 2009; G.E. DEMACOPOULOS, Gregory the Great. Ascetic, pastor and first man of Rome, Notre Dame, Indiana, 2015.

${ }^{2}$ Su queste posizioni, G. JENAL, In cerca di ordine quando l'apocalisse sembra vicina: Gregorio Magno e il monachesimo del suo tempo, in Gregorio Magno nel XIV centenario della morte, Roma, 2004 (Atti dei Convegni Lincei, 209), p. 245.

${ }^{3}$ R. GILLET, Spiritualité et place du moine dans l'Église selon Saint Grégoire le Grand, in Théologie de la vie monastique. Études sur la tradition patristique (= Théologie, 49), Paris, 1961, p. 321-351, in particolare p. 333.

4 È appena il caso di ricordare che i vescovi avevano avuta assegnata la responsabilità di controllare gli istituti monastici, specie per quanto attiene alla disciplina (già il Concilium Chalcedonensis, a. 451, in G. Alberigo et alii (a cura di), Conciliorum Oecumenicorum Decreta, Bologna, 1962, can. 8, p. 67; e il quinto Concilium Arelatensis, a. 554, in C. De Clercq (ed.), Concilia Galliae, a. 511-a. 595, Corpus Christianorum. Series latina [= CCSL], 148A, 1963, can. 2-3. 5, p. 171-172 si erano espressi in tal senso), ed erano anche chiamati a consacrare i nuovi monasteri o gli oratori: GELASIO, Epistulae, in A. Thiel (ed.), Epistulae romanorum pontificum genuinae, I, Brunsberg, 1868 (rist. Hildesheim-New York, 1974), XIV, 35, p. 375-376: De locorum consacratione sanctorum [...] absque praecepto sedis apostolicae nunnulli factas ecclesias vel oratoria sacrare praesumant; Liber Diurnus romanorum pontificum, ed. on line (= Liber Diurnus), cap. 5, p. 49 ss.; R. GRÉGOIRE, Strutture del potere e sistemi di governo nei monasteri benedettini medievali, in Inter fratres, 47, 1997, p. 1-25; e le puntualizzazioni in G. JENAL, op. cit. (n. 2), p. 236-240, 245. Sui doveri del vescovo, vd. l'analisi corredata dai riferimenti bibliografici e testuali, in C. URSO, Le donne al tempo di Gregorio Magno. La testimonianza del Registrum Epistularum, Trapani, 2013, n. 160, p. 73-74.

${ }^{5}$ Vigeva allora l'obbligo di permanenza, non ancora definitasi in clausura, nel chiostro. Sul tema, C. URSO, Donne di Sardegna nel Registrum Epistularum di Gregorio Magno, in L.G.G. Ricci (ed.), Gregorio Magno e la Sardegna, Convegno internazionale di studio (Sassari, 15-16 aprile 2005), Firenze, 2007, p. 182183 e n. 23 per la letteratura specifica; cfr. inoltre J. TIBBETTS SCHULENBURG, Strict active enclosure and its effects on the female monastic experience
} 
Dialoghi e, in particolare, nella presentazione del vir Dei Benedetto, non coincidono tout-court con i precetti della suddetta Regula . Ciò significa che «le comunità non appaiono sottoposte all'imperio della norma scritta, ma piuttosto all'autorità dell'abate, che governa in forza del suo carisma, $\mathrm{o}$, in alcuni casi, del priore» ${ }^{7}$. Insomma, come ripete Georg Jenal, Gregorio era legato ai comandamenti conciliari e imperiali più che ai capitoli di una Regola ${ }^{8}$.

Gregorio fu dunque un "monaco" sui generis: abbracciò la vita monastica solo per un breve tratto della sua esistenza, non scrisse alcuna regola ma seguì con la dedizione di un pastore nonché di un accorto amministratore e organizzatore l'intero movimento, e di "amici" monaci si servì per raggiungere taluni dei suoi più ambiziosi obiettivi. Per oltrepassare tuttavia il semplice seppur fondamentale recupero di questi elementi, che risultano più o meno approfonditamente indagati dalla storiografia gregoriana e da noi stessi, intendiamo proporre una lettura del Registrum attenta a fare emergere non tanto i contenuti e i modi più alti della missione pastorale del pontefice, quanto le prospettive economiche, sociali nonché politiche dei suoi interventi in campo monastico. O meglio, si esaminerà l'impatto che tali provvedimenti gregoriani ebbero sulla Chiesa, sui suoi progetti e sui suoi interessi politici e patrimoniali da una parte, così come, dall'altra, sulla società. Si recupereranno dunque gli esiti dell'azione pontificia sull'incremento dell'edilizia monastica con i suoi substrati evergetici anche di altissimo livello, sulle specificità dell'impegno gestionale dei vertici monastici, compresi quelli femminili, che adulteravano talvolta con preoccupazioni mondane le responsabilità precipue della carica e compromettevano lo spirito contemplativo delle congregazioni, e sulla decadenza morale che, con la complicità colpevole anche delle più alte cariche ecclesiastiche, segnava viepiù il contesto. Un criterio siffatto potrebbe, a mio parere, consentire di rintracciare nell'azione di Gregorio gli aspetti e i caratteri di una consapevole "politica monastica”, ricca di reconditi significati e di inaspettate finalità, superando così il parere contrario che pure è stato autorevolmente formulato ${ }^{9}$.

Tale premessa era doverosa per dare conto dell'approccio specifico che guiderà il nostro esame delle epistulae, strutturato ovviamente in maniera tale da sviluppare la ricerca nelle sue diverse articolazioni. A partire dall'edilizia religiosa.

\section{PROGETTO EDILIZIO: MONASTERI, ORATORI E XENODOCHI}

I progetti volti alla realizzazione di monasteri o a dare ospitalità a gruppi monastici in grandi case private riadattate all'uso si moltiplicarono già dai primissimi tempi del pontificato gregoriano. Nuovi edifici monastici, nuovi oratori e nuovi xenodochia sono attestati a Roma, in Sicilia e nelle altre isole piccole e grandi del Mediterraneo, in Campania, nella Lunigiana, nel ravennate e nel territorio della Pentapoli, specie nelle attuali Marche (Ascoli, Fermo), Abruzzo (Teramo) e Romagna (Rimini) e, fuori dal patriarcato romano, in Gallia ealtrove ${ }^{10}$. Le iniziative, cospicue in termini numerici ${ }^{11}$, provenivano da ecclesiastici, fra i quali lo stesso pontefice, e con una frequenza talvolta sospetta anche da benefattori laici. Fra questi ultimi si distinsero sovrane e alti dignitari: in Gallia, fu la regina Brunechilde, in concorso con il vescovo Siagrio, a fare costruire nella città di Autun una chiesa in onore di san Martino con a capo il presbitero e abate Lupo, ma anche il monastero femminile di Santa Maria, affidato alla badessa Talasia, e uno xenodochium, cui fu preposto un altro presbitero e abate, Senatore. La stessa regina, assieme al nipote re di Burgundia Teoderico II, per queste fondazioni

(500-1000), in J.A. Nichols, L.T. Schank (ed. by), Distant echoes. Medieval religious women, Kalamazoo, Michigan, 1984, p. 51-86; J. LECLERCQ, Théorie et pratique de la clôture au Moyen Âge, in Les religieuses dans le cloître et dans le monde des origines à nos jours, Actes du Deuxième Colloque international du C.E.R.C.O.R. (Poitiers, 29 septembre-2 octobre 1988), Saint-Etienne, 1994, p. 471-477 e n. 4 per la bibliografia sul tema; M. KELLY, Reflection on the significance of Stabilitas and Conversatio morum in benedictine commitment, in The American Benedictine Review, 5, 3, 2004, p. 260-262; H. RÖCKELEIN, Hiérarchie, ordre et mobilité dans le monachisme féminin, in F. Bougard, D. Iogna-Pratt, R. Le Jan (éd.), Hiérarchie et stratification sociale dans l'Occident médiéval (400-1100), Turnhout, 2008, p. 214-216.

${ }^{6}$ G. CREMASCOLI, I viri Dei di Gregorio Magno fra tentazioni e prodigi, in A. Bartolomei Romagnoli, U. Paoli, P. Piatti (a cura di), Hagiologia. Studi per Réginald Grégoire, I, Fabriano, 2012, p. 659-675, in particolare p. 662-669; sul tema, ampiamente discusso dagli specialisti attraverso un'attenta analisi del Registrum così come dei Dialoghi, anche S. PRICOCO, Gregorio Magno e la tradizione monastica, in C. Leonardi (dir.), Gregorio Magno e le origini dell'Europa, Atti del Convegno internazionale (Firenze, 13-17 maggio 2006), Firenze, 2014, p. 63-88, in particolare n. 3, p. 64 per la bibliografia più accreditata; A. DE VOGÜÉ, Le monachisme vu par Grégoire le Grand, ivi, p. 159-169. Per la discussione storiografica sul punto, C. URSO, op. cit. (n. 4), p. 28-31, n. 61, 64. ${ }^{7}$ La conclusione di S. PRICOCO, op. cit. (n. 6), p. 72, commenta per la verità i dati che emergono dai Dialoghi di Gregorio Magno, ma non mi pare forzato ampliarla fino a farne una costante dell'organizzazione monastica del tempo. Il concetto era già stato enucleato da R.A. MARKUS, op. cit. (n. 1), p. 68-69. La libera elezione dell'abate, altro elemento "benedettino" del monachesimo d'età gregoriana, è attestata in GREGORIO MAGNO, Registrum Epistularum, ed. D. Norberg, CCSL, 140-140A, 1982 (= GREG. Registrum), V, 49, a. 595; XIII, 10, a. 602.

${ }^{8}$ G. JENAL, Gregor der Große und das abendländische Mönchtum seiner Zeit, in Gregorio Magno e le origini dell'Europa, op. cit. (n. 6), p. 143-157. Sui caratteri del monachesimo nell'Occidente gregoriano, cfr., per un primo approccio, G. PENCO, Il concetto di monaco e di vita monastica in Occidente nel secolo VI, in Studia monastica, 1, 1959, p. 7-50; ID., Storia del monachesimo in Italia dalle origini alla fine del Medio Evo, Roma, 1961, p. 85-10o; ID., Condizioni e correnti del monachesimo in Italia nel secolo VI, in Benedictina, 27, 1980, p. 91-107; G. PICASSO, Il monachesimo occidentale dalle origini al secolo X, in G. Pugliese Carratelli (pref.), Dall'eremo al cenobio. La civiltà monastica in Italia dalle origini all'età di Dante, Milano, 1987, p. 8-16; G. JENAL, Italia ascetica atque monastica. Das Asketen- und Mönchtum in Italien von den Anfängen bis zur Zeit der Langobarden (ca. 150/250-604), I, Stuttgart, 1995, p. 726-748; B. MÜLLER, Gregory the Great and monasticism, in B. Neil, M. Dal Santo (ed. by), A Companion to Gregory the Great, Leiden-Boston, 2013, p. 83-108.

9 Per G. JENAL (op. cit. [n. 2], p. 245-246; Italia ascetica atque monastica. Das Asketen- und Mönchtum in Italien, op. cit. [n. 8], p. 309 ss.), non si può attribuire a Gregorio nessuna vera "politica monastica". Egli non mirava infatti «alla diffusione di una particolare forma ascetica o di una particolare regola [...]. L'ordine, all'interno e all'esterno, era lo scopo che si prefiggeva, quale premessa di una vita il più possibile tranquilla».

${ }^{10}$ Sulla dislocazione delle strutture monastiche considerate a vario titolo nell'Epistolario, di un certo interesse risultano le osservazioni, volte a ricordare che molte aree dell'Italia, e specificatamente quelle sottoposte al dominio longobardo, «si presentano [...] come una macchia bianca sulla carta, per quel che riguarda il fenomeno ascetico e monastico», di G. JENAL, op. cit. (n. 2), p. 221-222.

"Senza però raggiungere le cifre indicate da B. MÜLLER, op. cit. (n. 8), p. 95, dove la studiosa, certo confondendo il numero delle monache presenti a Roma nel 597 con quello delle istituzioni monastiche, scrive: «According to a statement by Gregory at June 597, he had the care of 3000 monastic institutions». 
chiese e ottenne da Roma speciali e generosi privilegi che sembrano anticipare almeno in parte la condizione propria delle abbazie nullius. Nessuna autorità laica o ecclesiastica, infatti, poteva usurpare i beni consegnati dai fondatori ai suddetti istituti; l'elezione dell'abate e della badessa era decisa dal re con l'assenso della comunità; la carica abbaziale non doveva essere oggetto di compravendita e la relativa giurisdizione era assegnata al vescovo di Autun coadiuvato da altri sei coepiscopi; nessun abate, infine, aveva la prerogativa di ricoprire la carica vescovile, avocando a sé gli averi dell'ente $^{12}$.

Politica monastica e politica generale del papato coincidevano: Gregorio, infatti, aveva allora in mente di servirsi anche della potente regina d'Austrasia Brunechilde, verso la quale perciò mostrava tanto interesse, per dare corpo ad un solido programma riformatore della Chiesa franca che, a sua volta, si iscriveva in un ambizioso progetto egemonico dell'autorità ecclesiastica romana sull'intero Occidente. Il trattamento speciale riservato alle fondazioni di Autun rientrava in questo disegno e, inoltre, concorreva a sottolineare la rilevanza politica della stessa civitas che, posta alla frontiera tra la Burgundia e l'Austrasia riunificate sotto il regno del figlio di Brunechilde, Childeberto II, era divenuta centro nevralgico, più strategico di Arles sede del vicariato apostolico ma ormai periferica rispetto al cuore pulsante dei regna merovingi. Insomma, Gregorio si collocava al fianco dei reges, della regina e del suo entourage ecclesiastico, perché nutriva serie speranze sulla reciproca volontà di riportare la chiesa locale ai livelli organizzativi e morali auspicati. In tale quadro, non è da sottovalutare il fatto che per Siagrio di Autun Brunechilde aveva chiesto nel 597 l'attribuzione del pallium. E Gregorio glielo aveva accordato non senza però avere fatto pesare con ritardi e relative vaghe delucidazioni la sua accondiscendenza, nel tentativo di approfittare della situazione per spronare, o forse è meglio dire costringere, i suoi interlocutori a seguire con più entusiasmo le spinte riformatrici'is.

Anche il monastero di San Cassiano a Marsiglia fu segnato a quel tempo da prestigiose novità e anche in questo caso si possono scorgere atteggiamenti particolarmente benevoli da parte della curia romana. A volerlo ampliare in maniera tale che fosse direttamente collegato, iunctis aedificiis, alla loro abitazione erano stati infatti il patrizio Dinamio e la moglie Aureliana (o Aurelia). Nell'ottobre del 596 Gregorio lo ricordava in una lettera indirizzata alla badessa pro tempore Respecta, cui confermava le concessioni a suo tempo elargite, in virtù delle quali, alla morte della badessa, unica responsabile della gestione patrimoniale, erano le stesse religiose a scegliere la subentrante senza interferenze esterne. Il vescovo, cui continuava a spettare la vigilanza sulla condotta delle sorores, era chiamato semplicemente a constatare la dignitas della prescelta. Si delineava così un'autonomia dall'apparato diocesano, che nell'area provenzale vantava "antiche" tradizioni, ma che, allora, mirava probabilmente a premiare la scelta esistenziale di due maggiorenti della burocrazia locale i quali pare avessero loro stessi abbracciato la vita religiosa, gratificando la Chiesa con la vasta eco del loro esempio e, soprattutto, con il patrocinio concreto della fondazione ${ }^{14}$.

L'ampia casistica dei "benefattori" annovera però anche personaggi meno noti le cui imprese nel settore alimentano spesso la convinzione che l'evergetismo aristocratico, che sostiene e spiega il richiamo verso le pratiche religiose, segnali, più che un rinnovamento spirituale, «una moda che nella realtà è un disorientamento spirituale», un mezzo per sfuggire ai problemi quotidiani sempre più angosciosi, e, in alcuni casi, un vantaggioso programma imprenditoriale ${ }^{15}$. Gregorio, come cercheremo di documentare, da una parte appoggia il movimento e si dimostra pronto a rispondere a tutte le questioni che vengono sottoposte al suo giudizio da sanctimoniales e monachi, così come da religiosae femina ${ }^{16}$, vescovi, defensores e pubblici ufficiali, dall'altra non esita a denunciare ogni atto e ogni comportamento dubbi, coinvolgendo nelle conseguenti operazioni le autorità diocesane. Pare insomma perfettamente consapevole dei rischi connessi a questa dimensione del fenomeno ma risoluto a mantenerlo nel solco dettato dalla religio e dalla lex, nonché, in ultima analisi, a piegarlo agli interessi della Chiesa.

L'excursus epistolare lo certifica. Nel giugno del 591, Gregorio, in risposta ad una missiva del defensor della Chiesa in Corsica, Simmaco, al suo segretario Bonifacio, si occupava per la prima volta di edilizia monastica e lo faceva a partire da un territorio insulare che doveva essere stato fino a quel momento privo di strutture monastiche, se egli stesso dichiarava che «quell'isola [...] non ha avuto fino ad oggi un

\footnotetext{
${ }^{12}$ GREG. Registrum, XIII, 5. 9-11, a. 602. Sui poteri e sulle funzioni delle badesse, sui privilegi che anticipavano l'istituzione delle abbazie nullius, vd. i dati testuali e bibliografici in C. URSO, op. cit. (n. 4), p. 80-9o e n. 181, 184, 189 (contra: G. JENAL, Gregor der Große und das abendländische Mönchtum seiner Zeit, op. cit. [n. 8], p. 146-148, dove si data l'inizio dei privilegi non prima del 628; e anche ID., Italia ascetica atque monastica. Das Asketen- und Mönchtum in Italien, op. cit. [n. 8], p. 733 e n. 51).

${ }^{13}$ Per un approccio generale al tema, è utile la bibliografia citata in C. URSO, op. cit. (n. 4), n. 360-361 di p. 152-153 e passim; vd. inoltre p. 196-208 sulla regina Brunechilde e le speranze che su di lei aveva riposto il pontefice; p. 84-87, 203-204 e n. 483 per i dati su Autun, su Siagrio e le fondazioni cittadine. Sui rapporti fra Roma e le corti franche, in particolare con la regina Brunechilde, cfr. di recente anche C. RICCI, Gregory's missions to the barbarians, in A Companion to Gregory the Great, op. cit. (n. 8), p. 34-46; L. PIETRI, Grégoire le Grand et les souverains du «Regnum Francorum», in Gregorio Magno e le origini dell'Europa, op. cit. (n. 6), p. 191-204, in particolare p. 192-194.

${ }^{14}$ GREG. Registrum, VII, 12, a. 596; e VII, 33, a. 597, sulla conversione religiosa della coppia. Sui due personaggi, C. URSO, op. cit. (n. 4), p. 40-41, n. 83. ${ }^{15}$ A.M. GIUNTELLA, Contributo ad una carta topografica dei monasteri d'Italia nell'età di Gregorio Magno: il caso di Roma, in L. Ermini Pani (a cura di), L'Orbis christianus antiquus di Gregorio Magno, Convegno di studi (Roma, 26-28 ottobre 2004), I, Roma, 2007, p. 88-9o (il saggio ripete la relazione presentata alla XLVIII Settimana di Spoleto che pertanto non è qui citata); F.R. STASOLLA, Modi e luoghi dell'assistenza nelle opere di Gregorio Magno, ivi, p. 245. ${ }^{16}$ L'espressione era «riferita di solito a pie signore interessate alla fondazione o alla conduzione di monasteri senza essere monache» (R. TURTAS, La cura animarum in Sardegna e Corsica dall'Epistolario di Gregorio Magno, in L. Casula, G. Mele, A. Piras [a cura di], Per longa maris intervalla. Gregorio Magno e l'Occidente mediterraneo fra tardoantico e altomedioevo, Atti del Convegno internazionale di studi [Cagliari, 17-18 dicembre 2004], Cagliari, 2006, p. 404), tanto è vero che mantenevano la proprietà sui loro beni, cosa che non era consentita a chi si monacava, come vedremo analizzando più avanti il caso di Siagria. Vd. intanto per i riferimenti legislativi, Novellae, in R. Schoell, G. Kroll (edd.), Corpus iuris civilis, III, Hildescheim, 1988, 5, 5; 76; 123, 38, p. 32-33, $379-381,621$.
} 
monastero». A compromettere fino a tal punto la progettazione religiosa in Corsica potrebbero essere stati i tentativi espansionistici longobardi nel Mediterraneo; certo è che il papa chiedeva la fondazione di un cenobio nuovo di zecca. Gli si era proposto, per la verità, di ripristinare un vecchio edificio, eretto, ma forse mai utilizzato, per volontà della religiosa femina Labinia e già «pronto a ricevere i monaci». Egli si mostrò decisamente ostile all'offerta e diede mandato a Simmaco di individuare, con la collaborazione dell'abate Orosio appositamente inviato da Roma, un luogo inaccessibile, a picco sul mare e fortificabile, un sito cioè tanto sicuro da potere resistere ad ogni aggressione. Né spaventava il papa l'eventualità che il terreno fosse di un privato: «Siamo disposti», scriveva, «a pagare il prezzo dovuto, pur di concludere qualcosa di determinato». Gli sviluppi del piano purtroppo non si lasciano cogliere appieno; anzi, non si ha nemmeno la certezza che esso abbia avuto un seguito ${ }^{17}$.

Nello stesso periodo - siamo nel luglio del 591 - Gregorio si era impegnato a fare erigere in Sicilia un altro monastero, detto Praetoritanus, ma ancora alla data della lettera del luglio-agosto dell'anno successivo al rector dell'isola Pietro lo stabile non era stato completato. La collera di Gregorio è fin troppo evidente, anche perché egli aveva già deliberato che al suddetto monastero fosse accostato un oratorio. «Da questo», scriveva con ironia al suo interlocutore, «non possiamo fare altro se non lodare l'interessamento della tua esperienza. Ma almeno adesso che sei stato sollecitato, svegliati e mostra quello che sei capace di fare in tale costruzione. Io dissi di non dare a quei monaci niente in denaro, ma non ti ho proibito di fabbricare loro il monastero» ${ }^{18}$.

Ci siamo avvalsi per iniziare la nostra indagine di questi due esempi, non solo o non tanto per la loro primazia temporale, quanto piuttosto perché ci consentono di definire immediatamente i problemi principali con cui Gregorio dovette confrontarsi, pienamente cosciente che, onde scansare il perpetuarsi di incresciose situazioni, era indispensabile agire con tempestività, oltre che con gli appropriati strumenti legali. Spiccava specialmente l'urgenza di accertare non tanto le pie intenzioni dei singoli, bensì l'origine, la titolarità dei progetti e il rispetto degli obblighi di legge; di vagliare con attenzione cioè le iniziative e, infine, di seguirne gli avanzamenti.

Tra i tanti infatti che erano soliti esprimere in vita o per via testamentaria la decisione di fondare nuovi istituti, assicurando anche le necessarie risorse economiche, alcuni (o i loro rappresentanti o, ancora, gli esecutori testamentari), accampando speciose motivazioni, ne procrastinavano $\mathrm{i}$ lavori, incuranti della normativa che imponeva tempi certi e brevi ${ }^{19}$. In qualche occasione si era addirittura verificato che immobili adibiti a monasteri fossero in realtà usati come abitazioni di chierici e di laici, generando confusione e degrado ${ }^{20}$, e anche che dei "benefattori" mirassero a mantenere la proprietà del bene e soprattutto la gestione dello stesso e delle sue rendite. Così si regolò a Siracusa nel 599 Capitolina, quando dispose per via testamentaria un legato annuo tratto de eodem monasterio, a carico cioè della stessa fondazione, da devolvere all'abate Traiano ${ }^{21}$. Al contrario, capitava anche che si cominciasse a costruire un fabbricato monastico o assistenziale (xenodochium) senza che questo fosse stato effettivamente dotato di un patrimonio e di rendite a tutela della sua sopravvivenza.

L'evergetismo, in realtà, nascondeva insidie che destavano forti preoccupazioni negli ambienti della corte pontificia e nello stesso pontefice, il quale non perdeva occasione per richiamare tutti all'osservanza della normativa, pretendendo sempre il deposito di precise e articolate garanzie prima di concedere qualsiasi autorizzazione. Si chiariscono così le "strane" eapparentemente immotivate resistenze di Gregorio nel caso riguardante la Corsica e la sua dura reazione davanti ai ritardi nel completamento del monastero siciliano. Non si trattava infatti di episodi isolati bensì di casi diffusi di malcostume e di cattivo governo. Le procedure andavano affinate e imposte con scrupolo, onde evitare il moltiplicarsi degli inconvenienti e dei rischi, che mettevano a repentaglio le stesse istituzioni monastiche.

La clarissimae memoriae femina Alessandria, rimasta vedova, aveva, ad esempio, creato a Napoli il monastero dei Santi Erasmo, Massimo e Giuliana, e lo aveva splendidamente dotato, costituendolo suo erede e assegnandogli diversi beni, fra i quali la metà della tenuta Papirianense in Sicilia. Eppure nel 599, anni dopo la sua morte, l'abate Fusco era stato costretto a rappresentare presso Gregorio l'impossibilità di entrare in possesso del suddetto cespite, perché gli amministratori dello xenodochium palermitano di San Teodoro, detentori dell'altra metà, non si decidevano a ripartire le due quote. Le operazioni erano risultate nei fatti parecchio complicate dalla circostanza che il monastero napoletano vantava, nello stesso territorio palermitano, altri possedimenti in comune con lo xenodochio. L'abate, giunto nell'isola, era pronto ad accordarsi su questi ultimi, ma non intendeva rinunciare ad un lascito testamentario già aggiudicato alla fondatrice da un tale Pompeo, perché era in grado di documentare che esso, in octo unciis, vale a dire per i due terzi, apparteneva per diritto ereditario al suo ente ${ }^{22}$.

\footnotetext{
${ }_{17}$ GREG. Registrum, I, 50, a. 591. Si dicono certi che nessun monastero fu in effetti edificato sul territorio della Corsica in quel contesto temporale, D. ISTRIA, Ph. PERGOLA, Moines et monastères dans les îles des mers ligure et tyrrhénienne (Corse, Sardaigne, archipel toscan et archipel ligure), in Hortus artium medievalium, 19, 2013, p. 73-78, in particolare p. 75. Per la situazione in Corsica, vd. C. URSO, op. cit. (n. 4), p. 40 e n. 82.

${ }^{18}$ GREG. Registrum, I, 54, a. 591; II, 50, a. 592; per l'identificazione del monastero, vd. V. Recchia (a cura di), Opere di Gregorio Magno V, Lettere/1-4, Roma, 1996-1999 = GREG. Lettere/1, p. 230, n. 3. Sulla vita monastica nella Sicilia del tempo gregoriano, specialmente F. PRINZ, Papa Gregorio Magno, il monachesimo siciliano e dell'Italia meridionale, in S. Pricoco, F. Rizzo Nervo, T. Sardella (a cura di), Sicilia e Italia suburbicaria tra IV e VIII, Atti del Convegno di studi (Catania, 24-27 ottobre 1989), Soveria Mannelli (Cz), 1991, p. 7-20.

${ }^{19}$ GREG. Registrum, IV, 9, a. 593. I tempi d'attuazione dei legati testamentari, che, a seconda della tipologia della costruzione, andavano da uno a cinque anni, sono precisati in Codex Justinianus, in P. Krueger (ed.), Corpus iuris civilis, II, Hildescheim, 1989, I, 3, 45, p. 31; e I, 3, 28, p. 21-22; I, 4, 27, p. 44, sul ruolo ispettivo consegnato al vescovo; Novellae, 131, 10, p. 658-659.

${ }^{20}$ GREG. Registrum, V, 1, a. 594; il deplorevole utilizzo è attestato nel territorio ravennate.

${ }^{21}$ Ibidem, X, 1, a. 599.

${ }^{22}$ Ibidem, IX, 171, a. 599, inviata al defensor della pars siracusana, Romano. A proteggere gli interessi del monastero di Fusco a Napoli doveva essere il vescovo Fortunato (IX, 172, a. 599) e il defensor di Palermo Fantino era incaricato di restituire allo stesso abate codices et vela trafugati dal presbitero Costanzo e portati in Sicilia (IX, 173, a. 599).
} 
Comeè palese, le donazioni testamentarie non bastavano per assicurare ad una comunità monastica solidità economica; meglio era contare anche su risorse immediatamente fruibili. Ecco perché, prima ancora che il vescovo Decio, nel 599 a Marsala, potesse benedire il monastero femminile edificato a solo (vale a dire dalle fondamenta) in una sua casa dalla gloriosissima femina Adeodata, Gregorio, recuperando le disposizioni di Pelagio I accolte nel Liber diurnus ${ }^{23}$, pretendeva un'ispezione volta ad appurare l'inesistenza di cadaveri inumati nel terreno e la consegna secundum morem, in reditu, di «dieci solidi [...], liberi da tributi fiscali, tre schiavi (pueri), tre paia di buoi, altri schiavi (mancipia), che servano in monastero in numero di cinque, cavalle in numero di dieci, vacche in numero di dieci, pertiche di vigne in numero di quattro, pecore in numero di quaranta, et cetera $»^{24}$. A proposito di un istituto maschile realizzato in una sua proprietà dal diacono della chiesa di Ascoli, Proculo, il donativo reclamato consisteva in «una conduma, un paio di buoi, cinque capi di bestie da soma, venti capi tra capre e pecore, i due terzi del fondo Gressiano, ove sorge lo stesso monastero, i due terzi del fondo Staziano, l'intero fondo Paterno, dieci maiali, i letti, con l'allegazione agli atti municipali e il resto secondo il solito» ${ }^{25}$.

Sulla diversa composizione delle clausole - di queste e di altre non ancora citate, che dunque non si possono catalogare come mera reiterazione di formulae della cancelleria pontificia ma appaiono calibrate sul fatto concreto - esporremo più avanti la nostra ipotesi. Per il momento ci interessa evidenziare come si intuisca fra le righe che il progetto romano era con buone probabilità orientato a recuperare il controllo di sempre più estesi patrimoni immobiliari e fondiari e delle masse contadine che vi risiedevano. Gregorio perseguiva l'obiettivo senza dichiararlo, ma avanzando le sue richieste con insistenza, specie in occasione della consacrazione degli oratori ${ }^{26}$, e invocando con eguale tenacia il ricorso alle scritture gestisque municipalibus allegata comprovanti il passaggio di proprietà. Nel contempo, richiamandosi fra le righe ai suoi doveri di pastore attento alle necessità del gregge, dimostrava con gli esempi che, in assenza di quelle garanzie, le comunità rischiavano di sperimentare situazioni veramente difficili. Quando infatti fu il momento di dedicare al nome della beata Maria l'oratorio che si era stabilito di accostare al monastero siciliano detto Praetoritanus o Praecoritanus citato poc'anzi, per consentire che l'avvenimento si festeggiasse in grande stile con il coinvolgimento della popolazione locale, Gregorio fu obbligato a dare ordini al defensor Pietro affinché, computando tutto nel suo rendiconto, provvedesse a «distribuire ai poveri dieci solidi d'oro, trenta anfore di vino, duecento razioni di pane (annonae), due anfore d'olio, dodici montoni, cento galline ${ }^{27}$. Sicuramente l'istituto, che peraltro non era stato ancora completato, non godeva di sufficienti entrate e viveva in condizioni di estremo disagio.

La costanza con la quale da Roma non si smise mai di indicare i requisiti economici e giuridici cui era subordinato il permesso di costruire e poi di intestare ai santi nuove strutture, valeva chiaramente anche a scongiurare l'eventualità che la Chiesa e, nello specifico, le chiese locali fossero oberate da insostenibili carichi finanziari conseguenti al loro "fallimentare" avvio. Fu così che, quando l'illustris Timotea, dopo avere innalzato un oratorio in un suo fondo nella diocesi di Rimini, chiese di intitolarlo alla Santa Croce, il vescovo titolare, Castorio, fu abilitato ad attenersi ai propositi della donatrice solo si [...] nullum corpus ibidem constat esse humatum, percepta primitus donatione legitima, id est, totius facultatis eius, excepta familia, mobilem vel immobilem, seseque moventem uncias octo [cioè i due terzi del bene], retento sibi usufructu diebus vitae suae, gestisque municipalibus alligat ${ }^{28}$. E nell'ottobre del 597 al vescovo di Luni, Venanzio, promotore nella sua dimora di un monastero femminile in onore dei santi Pietro, Giovanni e Paolo, Erma e Sebastiano, prima di procedere all'intestazione dell'oratorio annesso, si chiese che, constatata la mancanza di sepolture in loco, destinasse alla fondazione ogni suo bene, vale a dire «un calice d'argento del peso di sei once, una patena d'argento di due libbre, due sindoni, una tovaglia per altare, dieci letti, venti utensili di bronzo, trenta utensili di ferro e, in proprietà rustica, il fondo Faboriano e Lumbricata per intero, fondo situato in territorio di Luni, più o meno a due miglia dalla città, lungo il fiume detto Macra, con due schiavi, e cioè Mauro e Giovanni, due paia di buoi, tutto allegato agli atti del municipio» ${ }^{29}$.

Al deposito di cespiti altrettanto consistenti fu vincolata la dedicazione dell'oratorio voluto dal comes castri di Te-

\footnotetext{
${ }_{23}^{23}$ Liber Diurnus, cap. 5, tit. 4, p. 50: Responsum oratorii dedicandi. Hac formula bis usus reperitur Pelagius I [...], Gregorius Magnus [...]; sul tema, cfr. già C. VIOLANTE, Le strutture organizzative della cura d'anime nelle campagne dell'Italia centrosettentrionale (secoli V-X), in Cristianizzazione ed organizzazione ecclesiastica delle campagne nell'alto Medioevo: espansione e resistenze, Spoleto, 1982 (Settimane di studio del Centro italiano di studi sull'Alto Medioevo, 28, 2), p. 1000 e n. $43-44$ di p. 993 per le fonti; sulle procedure, specialmente J.R. C. MARTYN, Formulae for the equipment of monasteries, convents, and oratories, in Medievalia et humanistica, 30, 2004, p. 115-122.

${ }^{24}$ GREG. Registrum, IX, 233, a. 599, e XI, 5, a. 6oo, sulle reliquie inviate per la consacrazione del monastero; su Adeodata, anche VIII, 34, a. 598; sul punto vd., tra i tanti, F. RIGANATI, Reliquie, reliquiari e cose sacre dal Registrum Epistularum Gregorii Magni, in L'Orbis christianus antiquus di Gregorio Magno, op. cit. (n. 15), p. 531-576, passim. Per le procedure, volte a dare ordine e a fissare precisi protocolli, si rimanda a C. LEYSER, The temptations of cult: roman martyr piety in the age of Gregory the Great, in Early Medieval Europe, 9, 3, 2000, p. $209-302$.

${ }_{25}$ GREG. Registrum, XIII, 16, a. 602.

${ }^{26}$ Diversi oratori, destinati per disposizione canonica a garantire ad una comunità il culto divino, furono costruiti a quel tempo (esempi sono in GREG. Registrum, V, 50, a. 595, sull'oratorio approntato a Napoli da Andrea, abate di San Martino; XI, 19, a. 6o1, su quello fondato propriis sumptibus dal patrizio siciliano Venanzio); non è chiaro se fossero strutture sempre a supporto di un monastero o di una chiesa oppure anche indipendenti. Di norma, sostiene P. PELLEGRINI, La costituzione di oratori privati nell'età di Gregorio Magno. Il caso di Aprutium, in M. Rotili (a cura di), Tardo Antico e Alto Medioevo. Filologia, storia, archeologia, arte, Napoli, 2009, p. 58-59, quelli innalzati per iniziativa privata si trovavano in campagna, mentre quelli annessi ai monasteri in ambiente cittadino.

${ }^{27}$ GREG. Registrum, I, 54, a. 591.

${ }^{28}$ Ibidem, II, 11, a. 592.

${ }^{29} \mathrm{Ibidem}$, VIII, 5, a. 597. Le sindoni erano «probabilmente usate per avvolgere i pani liturgici»: F. RIGANATI, op. cit. (n. 24), p. 572, e passim per le reliquie impiegate nella consacrazione di chiese, altari, monasteri e oratori.
} 
ramo, Anione, in onore di san Pietro: il vescovo di Fermo, Passivo, a quel tempo visitatore nella diocesi teramana, doveva infatti controllare che fossero stati debitamente registrati al municipio e trasferiti «piccoli fondi con un'abitazione (fundi campuli cum conduma una) e gli annessi per un colono, un paio di buoi addomesticati, due vacche, quattro libbre di argento, un letto a giacere, un gregge di quindici capi, due utensili di bronzo, cinque utensili di ferro, sei solidi a disposizione liberi da tributi fiscali» ${ }^{30}$. Possedimenti e rendite ancora più cospicui, sui quali ritorneremo più avanti, aveva assicurato al monastero realizzato a Roma presso le terme di Agrippa il presbitero Giovanni, dettando le sue ultime volontà ${ }^{31}$.

Ammontava invece a soli 3 solidi liberi a tributis fiscalibus, esentasse cioè, la donazione di cui sempre il vescovo Passivo era chiamato a sincerarsi prima di collocare devotamente le reliquie del martire Sabino nell'oratorio che il notaio della Chiesa Valeriano aveva allestito a sue spese in fundo Visiano iuris sui iuxta muros civitatis Firmanae ${ }^{32}$. 10 solidi netti bastarono nel 599 alla religiosa femina Gennara, che aveva istituito propriis sumptibus un oratorio da intestare ai santi Severino confessore e Giuliana martire nella sua tenuta di Furiana in territorio di Tindari, per ottenere che l'ordinario locale Benenato celebrasse la cerimonia e collocasse sul posto le reliquie che ella aveva ricevuto dal vescovo Fortunato di Napoli33.

Resta da rimarcare, anche se pare ovvio da quanto fin qui riportato, che, affinché le procedure suddette fossero osservate e il fenomeno rimanesse confinato sotto il controllo diocesano e romano, non era tollerata la costruzione, né tantomeno la consacrazione di un edificio sacro sine praecepti auctoritate. Non a caso, si condannò con grande rigore l'operato irregolare di quidam episcopus Esusperanzio, che aveva eretto un oratorio nella diocesi del vescovo ravennate Giovanni senza prima avere chiesto e ottenuto le necessarie approvazioni, e si predispose l'immediata consegna del manufatto all'autorità locale ${ }^{34}$.

Quaestiones ancora più intricate e intriganti scaturivano dalle trasformazioni in complessi destinati all'alloggio di comunità monastiche di abitazioni preesistenti eappartenenti a benefattori laici, i quali appaiono troppo spesso intenzionati, come si è già accennato, a mantenerne il controllo.

Quest'ultima tipologia di insediamento monastico, che certo presupponeva pesanti interventi strutturali negli immobili prescelti, specie negli ambienti termali divenuti poco funzionali al nuovo utilizzo ${ }^{35}$, pare fosse molto ricorrente nelle isole dell'arcipelago ligure-toscano. Qui, come anche recentemente hanno ribadito Daniel Istria e Philippe Pergola, le indagini archeologiche hanno permesso «d'exhumer sur certaines d'entre elles les vestiges de villae somptueuses ou de structures très élaborées. Abandonnés à la fin de l'Antiquité, ces étabilissements constituèrent probablement des centres d'ancrage des premiers moines sur ces territoires alors peu ou pas habités» ${ }^{36}$. Ma gli esempi più noti, anche per le complicanze che Gregorio fu chiamato a sbrogliare, riguardano la Sardegna ${ }^{37}$.

Nella sua casa di Cagliari aveva deciso di riunire le consorelle la religiosa femina sarda Pompeiana, le cui vicissitudini, che si snodano per un lungo lasso di tempo dal 591 al 603, risultano ingarbugliate dalla sua contemporanea e attiva partecipazione al perfezionamento del progetto del defunto genero Epifanio, anch'egli desideroso di convertire la sua residenza in un monastero maschile. Le opposizioni parentali che ritardarono l'esecuzione degli atti testamentari di Epifanio, la "pericolosa” vicinanza fra le due strutture monastiche che fu considerata un possibile motivo di scandalo, assieme all'insipienza gestionale del vescovo locale Gianuario, incapace di mettere in pratica i suggerimenti pontifici, allungarono a dismisura i tempi di attuazione, tanto che non siamo in grado di accertarli $3^{8}$.

Sempre in Sardegna si registrano, nel 593, le peripezie di Teodosia, un'altra religiosa femina impegnata nell'adempimento delle decisioni testamentarie del marito Stefano che le aveva indicato anche il luogo, in praedio quod appellatur Piscenas, in cui collocare un nuovo monastero. La donna si era presto accorta - almeno così scriveva al papa - che impedimenti divenuti insormontabili non le avrebbero consentito di rispettare alla lettera i propositi del coniuge e aveva perciò proposto di sfruttarne il lascito per collocare una congregazione femminile in una sua dimora di Cagliari. I tanti ostacoli frapposti da non meglio individuati hospes e

\footnotetext{
30 GREG. Registrum, IX, 72, a. 598.

${ }^{31}$ Ibidem, VI, 44, a. 596; e infra, n. 46, 71.

${ }^{32}$ Ibidem, IX, 58-59, a. 598.

33 Ibidem, IX, 39, a. 598; 181-182, a. 599. Sulla questione nelle sue sfaccettature, sull'identificazione della protagonista e sui diritti che vantava sul fondo, C. URSO, op. cit. (n. 4), p. 48-50.

${ }^{34}$ GREG. Registrum, XIII, 17, a. 603.

35 P.G. SPANU, La Sardegna bizantina tra VI e VII secolo, Oristano, 1998, p. 201.

${ }^{36}$ Sul punto, vd. D. ISTRIA, PH. PERGOLA, op. cit. (n. 17), p. 73; cfr. lo studio sugli esordi, già dal secolo IV, del monachesimo insulare di J. BIARNE, Le monachisme dans les îles de la Méditerranée nord-occidentale, in Rivista di archeologia cristiana, 76, 2000, p. 351-374.

${ }^{37}$ Sul monachesimo sardo, che, se si eccettua l'esperienza di Fulgenzio di Ruspe agli inizi del secolo VI (R. TURTAS, Note sul monachesimo in Sardegna tra Fulgenzio e Gregorio Magno, in Rivista di storia della Chiesa in Italia, 41, 1978, p. 105-107; e i riferimenti bibliografici in D. ISTRIA, PH. PERGOLA, op. cit. [n. 17], p. 74-75 e n. 9-11 di p. 78), era specialmente cittadino e cagliaritano; cfr. G. JENAL, Italia ascetica atque monastica. Das Asketen- und Mönchtum in Italien, op. cit. [n. 8], p. 300-302; R. TURTAS, Gregorio Magno e la Sardegna: gli informatori del pontefice, in A. Mastino et alii (a cura di), La Sardegna paleocristiana tra Eusebio e Gregorio Magno, Atti del Convegno nazionale di studi (Cagliari, 10-12 ottobre 1996), Cagliari, 1999, p. 497-513; R. MARTORELLI, Gregorio Magno e il fenomeno monastico a Cagliari agli esordi del VII secolo, in Per longa maris intervalla, op. cit. (n. 16), p. 125-158; M.G. SANNA, L'epistolario sardo-corso di Gregorio Magno, in Gregorio Magno e la Sardegna, op. cit. (n. 5), p. 69-116; PH. PERGOLA, Christianisation, monastères et territoire en Sardaigne et en Corse, in Rivista di archeologia cristiana, 84, 2008, p. 365-376. Sulle protagoniste femminili del monachesimo sardo d'età gregoriana, nonché sulle incapacità gestionali del vescovo di Cagliari Gianuario, C. URSO, op. cit. (n. 4), p. 36-40.

${ }^{38}$ Su Pompeiana, GREG. Registrum, I, 46, a. 591; I, 61, a. 591; III, 36, a. 593, qui Pompeiana è citata, assieme ad un'altra religiosa femina, tale Teodosia, per fatti non del tutto chiari, probabilmente di natura morale, che le avevano coinvolte in un caso giudiziario che Gregorio aveva avocato a sé a Roma; XI, 13, a. 6oo; XIV, 2, a. 603, qui la donna è detta Pomponiana, ma altri dati presenti nella lettera ci inducono a considerarla omonima della precedente religiosa.
} 
supervenientes, sempre a suo dire, avevano bloccato il piano e per questo ella si era appellata a Roma. Gregorio, convintosi che potesse essere risolutiva la mediazione del vescovo Gianuario, gli conferiva il compito di sostenerla nel completamento dell'impresa e nella collocazione in loco delle reliquie che la donna già possedeva. Qualcosa tuttavia negli sviluppi degli eventi non convinceva. Forse, si sospettava, proprio al fine di spostare la fondazione nella sua abitazione, era stata la stessa Teodosia ad accampare dei pretesti per rinviare $\mathrm{i}$ lavori. Attorno al progetto era successo di tutto, precisava il pontefice nel 594, allorquando, nella speranza di chiudere in maniera dignitosa la quaestio, decideva di sottrarre la cura del monastero a Teodosia e di affidarlo ad un abate noto per la corretta osservanza delle regole ${ }^{39}$.

A Roma, da una casa privata della defunta patrizia Campana, si era ricavato un monastero femminile, che Gregorio aveva deciso di trasferire proprietatis iure proculdubio ad una badessa di nome Flora. Lo stabile era ubicato nella quarta regio, presso il Quirinale (oppure «sulle pendici sud-occidentali del Viminale, approssimativamente fra San Lorenzo in Fonte e San Lorenzo in Panisperna»), iuxta locum qui appellatur Gallinas albas, così come l'orto e gli alloggi già esistenti dentro il suo perimetro. La comunità aveva così in dotazione pertinenze utili a darle solidità economica ${ }^{40}$.

A Napoli, era stata l'illustris femina Rustica o Rusticiana ${ }^{41}$, consorte di Felice, a dettare nel suo testamento chiare istruzioni affinché nella sua dimora fosse eretto un monastero femminile per la cui guida era stata scelta la badessa Graziosa. L'edificio si trovava in regione Herculensi, in vico qui appellatur Lampadi; accanto era stato aggiunto anche un oratorio, dotato dalla nobildonna di un terzo dei suoi averi. La badessa si era rivolta al pontefice affinché autorizzasse la dedicazione del monastero e dell'oratorio alla Vergine Maria. Nella lettera dell'agosto 593, si incaricava il vescovo napoletano Fortunato di soddisfare la suddetta richiesta, non prima di avere appurato che tutto fosse giuridicamente definito. Il vescovo era invitato cioè a prendere visione del testamento per avere contezza della legalità dell'atto e dunque della donazione di Rusticiana ${ }^{42}$. Non pochi però furono gli intralci e i problemi che negli anni angustiarono i vertici dell'istituto, impossibilitati a dipanarli nonostante i continui suggerimenti dell'autorità pontificia. Sappiamo, infatti, da una lettera del novembre $598^{43}$ che la nuova badessa, Tecla, dovette affrontare un annoso e complesso procedimento giudiziario incardinato dal vir magnificus Alessandro. Ma c'è di più. Ricostruendo nei particolari i contatti fra la corte pontificia e i vari protagonisti dell'intera vicenda - Rustica, il marito Felice, il genero di quest'ultimo Alessandro, appena citato, e le badesse pro tempore, Graziosa e Tecla - emerge che la patrizia aveva deciso di innalzare un altro monastero in Sicilia. L'esecuzione di queste ulteriori disposizioni, che risalivano a più di venti anni addietro, era stata invece rimandata per ragioni imputabili allo stesso consorte Felice e ad Alessandro. Si era insomma corso il rischio che venissero intaccati i diritti della Chiesa cui Rustica aveva intestato diverse proprietà e che, soprattutto, era stata eletta esecutrice testamentaria ${ }^{44}$.

Ancora una volta appaiono chiari i motivi che giustificano l'estrema prudenza, quando non la diffidenza, con cui Gregorio fu solito trattare ciascuna di queste iniziative. Troppo spesso fu obbligato a risolvere gli esiti di operazioni dubbie, che tradivano in più di qualche caso l'insufficienza o lo storno di fondi. «Quanto al monastero del quale si dice che il defunto Pietro abbia ordinato l'edificazione nella sua casa», scriveva Gregorio a Gianuario di Cagliari nel settembre 593, «abbiamo stabilito che la fraternità tua esamini attentamente la quantità dei redditi rimasti. E se - raccolto tutto ciò che di quei beni si dice assottigliato o disperso - si ottiene una quantità sufficiente di tali beni, questo monastero sia fondato con ogni cura e senza alcuna dilazione. Se poi le possibilità sono scarse, o se si tratta di beni che comportano aggravi, [...] vogliamo che ti ci metta al corrente, in modo che sappiamo quello che [...] si possa deliberare su questa costruzione» 45 .

In mancanza di attente precauzioni e della corretta sorveglianza sui responsabili dell'attuazione degli atti testamentari, insomma, ogni progetto poteva essere vanificato: nella città di Pietro, ci informa una lettera del 596 indirizzata ad un abate non meglio identificato, era stato un presbitero, Giovanni, a volere ubicare nella sua abitazione sita presso le Terme di Agrippa un oratorio, a sua volta collegato ad un cenobio con annesso orto. Aveva in tal senso inserito nel suo testamento precise clausole che garantivano alla congregazione monastica che vi si sarebbe riunita reditus legati titulo. Tutto era stato impostato correttamente. Senonché, amministratori della Chiesa romana non solo non avevano dato seguito ai desiderata del presbitero, ma continuavano a gestire in proprio quei cespiti. Gregorio non intendeva indugiare. Decretava che l'oratorio fosse solennemente benedetto e che nel monastero fosse trasferita una familia di monaci provenienti da una struttura fatiscente cui potessero giovare i beni testati. Oltre all'orto, infatti, l'istituto possedeva una massa detta Magulianensis, grosso modo all'undicesimo miglio della via Nomentana, con tutte le sue dipendenze e, sempre a Roma, una taberna vicino al Pallacene e un salgamum - una dispensa cioè o una cantina, oppure una bottega

\footnotetext{
39 Ibidem, III, 36, a. 593; IV, 8. 10, a. 593; V, 2, a. 594.

${ }^{40} \mathrm{Ibidem}$, III, 17, a. 593. Sulla collocazione del manufatto sulle pendici del Quirinale o del Viminale nella quarta regio, vd., per la prima indicazione, GREG. Lettere/1, p. 405, n. 2; S. F. WEMPLE, Couvents de femmes en Italie, de lépoque du pape Grégoire le Grand aux environs de goo, in Les religieuses dans le cloître, op. cit. (n. 6), p. 76; per la seconda, A. M. GIUNTELLA, op. cit. (n.15), p. 90 (sua è la citazione nel testo); O. BUCARELLI, La carità del papa. Benefici e beneficiati nelle Epistole di Gregorio Magno, in L'Orbis christianus antiquus di Gregorio Magno, op. cit. (n. 15), n. 51-52 di p. 429, ove bibliografia sul monastero. Sulla patrizia Campana (e non della Campania, come in GREG. Lettere/1, p. 405), C. URSO, op. cit. (n. 4), p. 51, n. 107.

${ }^{41}$ Sul personaggio e per una ricostruzione più puntuale dell'intera vicenda, C. URSO, op. cit. (n. 4), p. 160-166.

${ }^{42}$ GREG. Registrum, III, 58, a. 593. Un cenno sibillino alle capacità finanziarie della donna è in Ep. I, 42, a. 591. Sempre a Napoli, il vir spectabilis memoriae Romano trasformò la sua casa in un monastero (IX, 10, a. 598), la cui consacrazione, fatti salvi tutti i soliti requisiti, fu disposta da Gregorio nel giugno 599 (IX, 166).

${ }^{43}$ Ibidem, IX, 54, a. 598, su cui infra, n. 76 .

${ }^{44}$ Ibidem, IX, 165, a. 599.

${ }_{45}$ Ibidem, IV, 9, a. 593.
} 
di frutta e legumi - situato proprio davanti all'edificio ${ }^{46}$. Tre anni dopo, la questione era ancora aperta; Gregorio scriveva in un'altra lettera, destinata alla badessa Bona, di avere deciso, fallito il primo progetto, di passare a lei e alle sue sorores l'intera proprietà, comprensiva di oratorio, monastero, orto e di altri possessi ${ }^{47}$. Intanto però gli agenti della «nostra Chiesa» continuavano a mantenerne la gestione, e noi, privi di notizie sugli avvenimenti successivi, non sappiamo neanche se le ordinanze pontificie furono rispettate oppure rimasero inevase. E tanto basta.

\section{MONASTERI: LUOGHI DI PACE E DI RIPOSO?}

La vita nei monasteri del tempo gregoriano scorreva, dunque, in mezzo a tanti vincoli e a tante difficoltà. I problemi si sommavano e pretendevano un coinvolgimento individuale costante e faticoso, trasformando i chiostri da oasi di pace e di riposo in luoghi di duro lavoro e di aspri scontri. La situazione era particolarmente difficile nelle isole e ancora di più in quelle che componevano i piccoli arcipelaghi del Tirreno, se è vero che lo stesso Gregorio, nel giugno 591, non solo escludeva che dei giovani ante decem et octo annorum tempore potessero essere accettati in Palmaria aliisque insulis (cioè, oltre che nell'isoletta di Palmarola, anche nell'isola Eumorfiana, ovvero forse l'isola di Zannone ${ }^{48}$, e nelle altre del gruppo delle Isole di Ponza), ma voleva che quelli già presenti fossero inviati a Roma ${ }^{49}$.

Spesso dovettero essere i monaci a riparare, direttamente o per mezzo di maestranze che andavano comunque controllate, i danni delle loro "case", nonché in particolare a seguire i lavori di realizzazione e, alla bisogna, di sistemazione dei tetti. Nell'isola Eumorfiana appena citata, sempre nel 591, si autorizzava il prelievo dal deposito isolano e la consegna all'abate Felice di ben 1500 libbre di piombo, che con buone probabilità sarebbe stato impiegato per uno degli scopi appena suggeriti. L'impegno di spesa, probabilmente troppo onerosa per la comunità religiosa, alla fine dei lavori andava rubricato nel rendiconto del suddiacono Antemio che era il rettore del patrimonio campano ${ }^{50}$.

Ove e quando necessario, i monaci, così come i chierici e i laici, dovevano prestare servizio di guardia delle mura, con turni talvolta tanto gravosi da spingere molti a sottrarsi all'obbligo ${ }^{51}$. Tanto è vero che, in un'occasione, fu lo stesso Gregorio, evidentemente a conoscenza dell'impossibilità di soddisfare la domanda dell'autorità competente, a chiedere al magister militum della Campania Maurenzio di alleggerire, nel caso in cui non fosse stato possibile abolirle del tutto, le prestazioni spettanti a Teodosio, abate del monastero voluto dal patrizio Liberio nella regione campana ${ }^{52}$.

Le comunità monastiche erano inoltre coinvolte nello sfruttamento dei cespiti fondiari di cui disponevano già all'atto della loro costituzione, o che erano successivamente testati da benefattori ${ }^{53}$. E ci si riferisce qui alla messa in produzione dei fondi attraverso il lavoro manuale, più che alla "gestione" finanziaria, faccenda per la quale si auspicava che specialmente le istituzioni femminili - per motivi anche di ordine morale, come si vedrà a proposito di quelle sorores sarde costrette a girare per i campi con gravi esiti si avvalessero di procuratores, ovvero di amministratori di specchiata onestà e membri dell'ordo ecclesiasticus. A differenza delle chiese, «le cui rendite e beni sono amministrati dal vescovo, dal suo economo e dal clero [...] e sono perciò finanziati dai fondi centrali», i monasteri, gli oratori e i centri assistenziali, infatti, «traggono sostegno dalle offerte dei fedeli e dal proprio lavoro» ${ }^{54}$. E pertanto, nonostante che le strutture fossero fornite di manodopera servile, nelle fonti gregoriane si incontrano monaci intenti a eseguire vari lavori agricoli, mentre le monache, a causa della mollitia del loro genere, godevano di speciali riguardi ${ }^{55}$. Fra i monaci lavoratori descritti specialmente nei Dialoghi sono da ricordare, giusto per proporre alcuni esempi, Equizio, che usa la falce di solito appesa al suo collo per tagliare il grano nel campo del suo monastero, o quei fratres occupati in più località nella raccolta delle olive ${ }^{56}$.

$\overline{4^{6}} \mathrm{Ibidem}, \mathrm{VI}, 44$, a. 596. Sul termine salgamum, L. PANI ERMINI, Testimonianze archeologiche di monasteri a Roma nell'altomedioevo, in Archivio della Società romana di Storia patria, 104, 1981, p. 32-33; S.F. WEMPLE, op. cit. (n. 40), p. 78-79.

${ }^{47}$ GREG. Registrum, IX, 138, a. 599.

${ }^{48}$ Così in O. BUCARELLI, op. cit. (n. 4o), p. 427, n. 36.

${ }^{49}$ GREG. Registrum, I, 48, a. 591.

${ }^{50}$ Ibidem, I, 48. Per questi utilizzi del piombo, vd. S. DEL LUNGO, Il paesaggio e l'organizzazione agricola negli scritti di Gregorio Magno, in L'Orbis christianus antiquus di Gregorio Magno, op. cit. (n. 15), p. 313.

${ }^{51}$ GREG. Registrum, VIII, 19, a. 598, indirizzata al vescovo di Terracina, Agnello. Gregorio esorta il suo interlocutore a vegliare affinché nessuno eviti i turni di guardia, fondamentali per civitatis custodia; IX, 11, a. 598, al vescovo di Cagliari, affinché, nelle more che si sottoscriva la pace con i Longobardi, organizzi la difesa delle mura; IX, 240, a. 599, all'erogator Donnello in merito alla custodia delle mura di Roma; G. DAMIZIA, Lineamenti di diritto canonico nel "Registrum epistularum" di san Gregorio Magno, Roma, 1949, p. 63, n. 2.

${ }^{52}$ GREG. Registrum, IX, 163, a. 599. Per le notizie sull'abate Teodosio e sul monastero che guidava, vd. GREG. Lettere/3, p. 364-365, n. 2-3.

${ }^{53}$ A proposito delle ricchezze patrimoniali degli istituti monastici, secondo E. CALIRI (Società ed economia della Sicilia del VI secolo attraverso il Registrum Epistularum di Gregorio Magno, Messina, 1997, p. 73), «non ci è noto come fosse definita la posizione giuridica di un monastero o di uno xenodochio in qualità di proprietari e se vi fossero vincoli con la Chiesa romana». In realtà, l'impiego nelle lettere gregoriane che destinano beni ad istituti monastici e similari di espressioni quali iure proprietario o proprietatis iure proculdubio, incrina, a mio parere, l'opinione di quanti (vd. specialmente F. MARAZZI, I "Patrimonia Sanctae Romanae Ecclesiae" nel Lazio [secoli IV-X]. Struttura amministrativa e prassi gestionale, Roma, 1988, in particolare p. 103-206; ID., I patrimoni della chiesa romana e l'amministrazione papale fra tarda antichità e alto medioevo, in P. Delogu [a cura di], Roma medievale. Aggiornamenti, Firenze, 1998, p. 46-47) ritengono che simili concessioni non intaccavano i diritti di proprietà della Chiesa in quanto essa non alienava i beni, ma li concedeva in uso; vd. l'approfondimento in C. URSO, op. cit. (n. 4), n. 114 di p. 53-54.

${ }^{54}$ Novellae, 120, 6, p. 582-285; P. PELLEGRINI, op. cit. (n. 26), p. 57.

55 GREGORIO MAGNO, Regola pastorale, in ID., Omelie sui Vangeli - Regola pastorale, a cura di G. Cremascoli, Torino, 1968, III, 1, p. 515; in tema di mollitia muliebre, vd. C. URSO, Tra essere e apparire. Il corpo della donna nell'Occidente medievale, Acireale-Roma, 2005, p. 25 ss.; H. RÖCKELEIN, op. cit. (n. 5), p. $205-206$.

${ }^{56}$ Nell'ordine di citazione: GREGORIO MAGNO, Dialoghi (I-IV), a cura di B. Calati, Suore Benedettine Isola San Giorgio, A. Stendardi, Roma, 20oo (= GREG. Dialoghi), I, IV, 13, p. 95; I, VII, 5, p. 107; vd. inoltre II, XXXII, 1, p. 199, sul falcetto usato da Benedetto per lavorare nei campi; III, XIV, 6, p. 247, sugli «attrezzi agricoli che volgarmente chiamiamo vanghe»; IV, XX, 2, p. 367, sull'abate Stefano che di sua mano aveva seminato un campo. 
Nel Registrum leggiamo invece che Gregorio accordò al monastero sito in Blerana civitate, presumibilmente nella Tuscia, due piccoli campi (una appendix quae Agellus dicitur e una terrula modiorum $X X X)$ in enfiteusi trentennale proprio per accogliere la richiesta specifica dei monaci, i quali de terris se suggesserunt necessitatem gravissimam sustinere. Ne avrebbero tratto solacium e avrebbero affrontato con i giusti mezzi il momento di difficoltàn7. Mosso da eguali finalità, il defensor campano Antonino aveva testato ad un monastero delle somme di danaro vincolate all'acquisto di terre ${ }^{58}$.

Ad ogni buon modo, è ora tempo di ritornare agli elenchi di quei beni e di quegli strumenti necessari, come abbiamo appurato sopra, per ratificare la fondazione di un istituto religioso, in cui "voci" costanti erano condumae, cioè fattorie ovvero «semplici edifici eretti negli appezzamenti o vero e proprio luogo di riferimento del fundus», e poi entrate in danaro, servi, coppie di buoi, giumenti, cavalle, e poi ancora ovini e caprini, maiali ecc. Ebbene, tali liste coincidono in maniera eloquente con «la dotazione di base per avviare la messa a coltura e lo sfruttamento di un terreno, compreso nell'elargizione»59. Pare inoltre plausibile avanzare l'ipotesi che, ove possibile, le assegnazioni si differenziassero nei contenuti a seconda del genere della congregazione cui erano destinate. A tutte le comunità servivano fondi, animali e rendite finanziarie. E tuttavia, quelle femminili necessitavano maggiormente di servi e serve, ovvero della manodopera che le consorelle non potevano prestare, quelle maschili specialmente di strumenti e animali. Certo non è una regola, ma resta significativa a tale proposito la richiesta fatta, ad esempio, per il monastero, femminile appunto, voluto da Adeodata a Marsala, richiesta nella quale ai solidi, alle pertiche di vigneto e ai diversi animali si aggiungevano servi e mancipia, rispettivamente «tre schiavi [...] e altri schiavi (mancipia), che servano in monastero in numero di cinque». Rappresentavano, riflette Domenico Vera, la forza-lavoro tipica di una "fattoria" siciliana del tempo che rendeva «al netto delle imposte 10 solidi annui» ${ }^{60}$.

Altrettanto interessante, per meglio definire lo scenario, è evidenziare che capi di bestiame erano inclusi tra i lasciti testamentari alla Chiesa. Nel 598, il diacono Servus-Dei, già amministratore in Sicilia dei patrimonia ecclesiastici al tempo di Pelagio II, aveva testato i suoi averi alla Chiesa; aveva però riservato, come legato speciale, quattro cavalle alla monaca Aluminosa, sua congiunta. Su richiesta del diacono Fiorenzo, Gregorio, attingendo allo stesso atto, ne conferiva altre dieci, con l'aggiunta di uno stallone per la riproduzione, allo xenodochium degli Anici di Roma, diretto allora dallo stesso Fiorenzo ${ }^{61}$. Ogni capo di bestiame costituiva, infatti, un cespite il cui valore «nella migliore delle condizioni, per esemplari di razza, può arrivare alla cifra di 12 aurei a capo ${ }^{62}$. Informato di ciò, Gregorio, volendo gestire con profitto, e non in perdita come avveniva allora in Sicilia, le mandrie di buoi e di cavalle possedute nell'isola, dopo avere deliberato che quattrocento cavalle fossero ripartite fra i conduttori di ogni singula condoma, decise di mettere in vendita gli altri esemplari63.

Comunque sia, seguire i lavori agricoli per mettere in produzione i terreni garantiva il rifornimento di abbondanti scorte di grano ${ }^{64}$, di olio, di vino, di miele, ecc. Si creavano le giuste premesse per risolvere le urgenze quotidiane delle comunità ma anche per poter distribuire viveri e beni di prima necessità agli indigenti. A soddisfare le stesse esigenze concorreva la proprietà o quantomeno la disponibilità di un hortus, inteso come un ad condimenta holerum nutrienda locus isdem aptus. Simili loci citati nelle lettere, e ancora di più nei Dialoghi ${ }^{65}$, erano spazi «di dimensioni medio-piccole, particolarmente diffusi nell'Alto Medioevo entro le città» nel cui paesaggio si erano riscontrati «fenomeni di ruralizzazione [...] nel trapasso dall'antichità al medioevo ${ }^{66}$. Qui monaci e monache producevano gran parte delle verdure, degli ortaggi e degli "odori" utilizzati nelle loro cucine. E si può anche ragionevolmente supporre che, per arricchire l'alimentazione con le loro carni e con le loro uova, si organizzassero negli horti, così come nei fundi di più ampia dimensione, pollai nei quali razzolavano galline e altri animali di piccolo taglio ${ }^{67}$.

Tali spazi potevano però servire anche come silenziosi e piacevoli luoghi in cui sostare per riposarsi o per meditare.

57 GREG. Registrum, IX, 97, a. 599. Sui termini usati per indicare i due fondi e specialmente per il toponimo Agellus, «preso in prestito direttamente dal frasario tecnico dei catasti», vd. S. DEL LUNGO, op. cit. (n. 50), p. 359.

${ }^{58}$ GREG. Registrum, IX, 106, a. 599.

${ }^{59}$ Le citazioni in S. DEL LUNGO, op. cit. (n. 50), p. 342-343. Per ciò che attiene all'organizzazione delle comunità monastiche in questo settore, vd. anche, seppur non sempre condivisibile nelle sue conclusioni (ad esempio quando sostiene che i monaci non dovevano occuparsi delle cose materiali cui avrebbe invece provveduto la Chiesa: p. 134), R. MARTORELLI, op. cit. (n. 37), p. 133-136.

${ }^{60}$ Per l'epistola gregoriana, supra n. 24; la citazione è di D. VERA, L'altra faccia della luna: la società contadina nella Sicilia di Gregorio Magno, in Studi storici, 47, 2, 2006, p. 448.

${ }^{61}$ GREG. Registrum, IX, 8, a. 598; sul diacono, I, 42, a. 591; XIII, 20, a. 603.

${ }^{62}$ S. DEL LUNGO, op. cit. (n. 50), p. 352; cfr. già V. RECCHIA, Gregorio Magno e la società agricola, Roma, 1978, p. 108, dove si ricorda il brano dei Dialoghi nel quale un vescovo distribuiva ai poveri i 12 solidi d'oro che aveva ricavato dalla vendita di un cavallo (GREG. Dialoghi, I, IX, 10, p. 115).

${ }_{63}$ GREG. Registrum, II, 50, a. 592.

${ }^{64} \mathrm{Il}$ riferimento al pagamento a rusticis ecclesiae delle imposte in natura, nello specifico in frumentum, attestato nello stesso epistolario (Ibidem, I, 42, a. 591) è d'obbligo.

${ }_{65}$ Per gli orti monastici: GREG. Dialoghi, I, III, 1-4, p. 85-87; I, IV, 7, p. 91; I, VII, 2, p. 105; II, VI, 1, p. 155; III, XIV, 6, p. 247; IV, XXIII, 1, p. 371. Sulle caratteristiche di questi spazi urbani «sia messi a coltivo che funzionali ad altre attività domestiche» [L. PANI ERMINI, Evoluzione urbana e forme di ruralizzazione, in Città e campagna nei secoli altomedievali, Spoleto, 2009 (Settimane di studio del Centro italiano di studi sull'Alto Medioevo, 56), p. 669-67o], vd. anche EAD., Forma urbis: lo spazio urbano tra VI e IX secolo, in Roma nell'alto medioevo, 1, Spoleto, 2001 (Settimane di studio del Centro italiano di studi sull'Alto Medioevo, 48), p. 260 sull'origine tardoantica degli orti romani; F. MARAZZI, Da suburbium a territorium: il rapporto tra Roma e il suo hinterland nel passaggio dall'antichità al medioevo, ivi, p. 737-740.

${ }^{66}$ Le due citazioni si debbono, nell'ordine, a S. DEL LUNGO, op. cit. (n. 5o), p. 348; L. ERMINI PANI, La Roma di Gregorio Magno, in L'Orbis christianus antiquus di Gregorio Magno, op. cit. (n. 15), p. 38.

${ }^{67}$ Centum gallinae erano elencate fra i doni ai poveri previsti in Sicilia in occasione della dedicazione di un oratorio: GREG. Registrum, I, 54, a. 591, e supra, n. 27. In S. DEL LUNGO, op. cit. (n. 50), p. 353, si precisa che il pollaio, in generale, è «ipotizzabile di varie dimensioni, a seconda della destinazione per la quale è stato concepito, ad uso commerciale o privato». 
Nell'incipit di una lettera del 593, Gregorio precisava che «Noi dobbiamo tenere in dovuta considerazione quelli che scelgono la vita religiosa, perché la necessità non li renda inoperosi e ne infranga - il che sia lungi - il vigore del genere di vita». Egli aveva allora disposto la trasformazione a Roma di un fabbricato privato in monastero e la consegna allo stesso di un orto e di altri alloggi compresi nella stessa area; aveva poi devoluto tutto alla badessa Flora e alle sue consorelle che ne avrebbero usufruito proprietatis iure proculdubio. L'intera comunità aveva in godimento una casa e un orto, non solo - ed è questo il punto - per fare fronte ai fabbisogni materiali ma anche per rinvigorire lo spirito ${ }^{68}$.

A Roma gli orti, nonostante il declino imputabile alle rovine delle invasioni barbariche, erano ancora numerosi ${ }^{69}$ : nell'epistola inviata al suddiacono Sabino tra il settembre e l'ottobre del 592, il pontefice ordinava che, sempre ne hii qui Dei (se) servitio deputasse noscuntur necessitatem aliquam possint [...] sustinere, l'orto già di un presbitero di nome Feliciano, sito nella regio prima di Roma ai piedi dell'Aventino, davanti alla scalinata di Santa Sabina, fosse dato alle monache del monastero di Euprepia ${ }^{70}$. Un altro orto, sempre a Roma, era annesso alla casa vicina alle terme di Agrippa, in cui il defunto presbitero Giovanni, che ne era il proprietario, aveva deciso di costruire un oratorio per accogliervi un gruppo di monaci, e che invece era poi stata affidata alla comunità femminile guidata dalla badessa Bona ${ }^{71}$. L'orto del monastero femminile di Santo Stefano, vicino alla basilica romana di San Paolo, era adiacente a «due orti posti tra il Tevere e i portici della stessa chiesa [...] divisi dal fiume Almon, tra i confinanti dell'orto del monastero di Santo Stefano [...] e i confinanti della tenuta Pisiniani». Questi ultimi due appezzamenti facevano parte della donazione ben più cospicua elargita alla basilica per provvedere all'illuminazione dell'edificio ${ }^{72}$.

Fundi, condumae, horti, vinae, terrae e quant'altro costituivano l'intero patrimonio fondiario degli istituti, messo a produzione grazie, come si è detto, al lavoro di manodopera servile e degli stessi religiosi ma controllato e talvolta gestito direttamente dagli abati e dalle badesse per, all'occorrenza, difenderlo da ogni attacco esterno ed interno. I tentativi di usurpazione c'erano ed erano talvolta molto insidiosi, se è vero che Gregorio, consapevole di quanto esposti fossero alla cupidigia degli uomini tali beni, concesse al monastero di Sant'Andrea al Clivus Scauri, da lui stesso fondato, un privilegio in base al quale si vietava a chiunque, ivi compresi l'abate, il vescovo e quicumque homo potens aut inferior, di alienare a terzi le proprietà della fondazione o di mettere in atto qualunque azione di appropriazione indebita ${ }^{73}$. Immediatamente dopo la sua elezione, peraltro, spinto dalle tante vertenze sottoposte al suo giudizio, si era premurato di definire i contorni dell'amministrazione patrimoniale in termini giuridici. Esemplare in tale contesto è la conduzione di una vicenda nella quale era stato coinvolto da un'interpellanza avanzatagli nell'ottobre 590 dal servus Dei Gregorio, presbitero nonché abate del monastero siciliano, o meglio palermitano, di San Teodoro. L'abate, più tardi accusato da Roma di avere guidato con lassismo la sua comunità, lamentava la sfrontatezza degli uomini di un fundus della Chiesa romana, detto "Fulloniaco", che avevano invaso i confini da tempo fissati di un altro podere, quello "di Gerdina", appartenente al suo monastero quieto iure per innumeros annos. Gregorio affidava la quaestio al suddiacono Pietro, amministratore del patrimonium Petri in Sicilia, ma gli indicava nel contempo la norma giuridica da applicare. Una Novella del Corpus giustinianeo, infatti, prevedeva la prescrizione quarantennale per i lasciti intestati a loci ecclesiastici. Dunque, si imponeva l'urgenza di stabilire tramite una mirata indagine se $\mathrm{i}$ fines fundi contesi fossero rimasti invariati veramente e ininterrottamente per quarant'anni o se al contrario ai monaci fosse stata mossa in quel lasso di tempo qualche contestazione. Ove si fosse verificata la seconda ipotesi, agli agenti addetti all'ispezione si suggeriva di depositare la contesa nelle mani di una commissione di arbitri appositamente eletti ${ }^{74}$.

A Gregorio, lo ribadiamo, sembrava di tutta evidenza che bastasse invocare la legge e applicarla per risolvere in trasparenza e legalità ogni lite. «La tua esperienza», aggiungeva infatti a chiusura della lettera, «porti a compimento tutte queste disposizioni in modo che, dopo ciò, una controversia del genere non ritorni a me». Ed invece i casi si moltiplicarono: nel 597 una grave disputa si scatenò tra l'abate del monastero di San Pietro ad Baias (Capo Miseno, Napoli) e quello di Santa Lucia in Siracusa. Ciascuno dei due abati imputava all'avversario di volere ridisegnare i confini di un fondo sito in Sicilia per ridimensionare l'estensione dell'altrui patrimonio. Fu deciso di affiancare all'ordinario locale un agrimensore appositamente giunto nell'isola da Roma, grazie alle cui competenze le due parti in causa, convinte dalla regolarità dei calcoli da quello effettuati, giungessero ad un accordo ${ }^{75}$.

Se l'impegno volto a ricomporre tutte queste questioni dovette farsi sempre più gravoso per la curia pontificia, al-

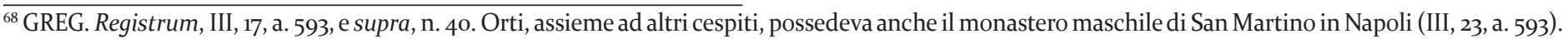

${ }^{69}$ Erano concentrati, si sostiene in S. DEL LUNGO, op. cit. (n. 50), p. 321-322, «nelle regiones degli Alta Semita (VI) e dell'Esquilinus (V)».

$7^{70}$ GREG. Registrum, II, 46, a. 592. Per la collocazione della costruzione «ai piedi dell'Aventino», vd. GREG. Lettere/1, p. 357, n. 2.

${ }^{71}$ GREG. Registrum, VI, 44, a. 596; IX, 138, a. 599; e per altri dettagli, supra n. 31, 46.

${ }_{72}$ Ibidem, XIV, 14, a. 604.

${ }^{73}$ Ibidem, Appendice II (1), a. 590-591, indirizzata all'abate pro tempore Massimo, poi vescovo di Siracusa. Sul problema più volte affrontato da Gregorio con riferimento alla proprietà ecclesiastica in generale, non solo monastica dunque, vd. S. DEL LUNGO, op. cit. (n. 50), p. 358-365 ss.; C. URSO, op. cit. (n. 4), p. 6o-64.

${ }^{74}$ GREG. Registrum, I, 9, a. 590; V, 4, a. 594; Novellae, 131, 6, p. 657. Sulle competenze giuridiche di Gregorio, si rimanda agli studi di A. PADOA-SCHIOPPA e, fra questi, specialmente a Il rispetto della legalità nelle Lettere di Gregorio Magno, in O. Condorelli (herausg.), Der Einfluss der Kanonistik auf die europäische Rechtskultur, Köln, 2009, p. 25-31.

75 GREG. Registrum, VII, 36, a. 597. Sui confini e sulle problematiche ad essi connesse, vd. in particolare S. DEL LUNGO, La pratica agrimensoria nella Tarda Antichità e nell'Alto Medioevo, Spoleto, 2004, passim, e in particolare p. 358 laddove si precisa che «La scelta [cioè di inviare un agrimensore romano] dipende probabilmente non dall'assenza nell'isola di persone professionalmente qualificate, ma dalla maggiore preparazione avuta dai tecnici formatisi a Roma, avendo ancora a disposizione l'intero patrimonio documentario conservato nei locali del Tabularium e ben lungi dall'essere gli ultimi rappresentanti di una categoria in via di sparizione».
} 
trettanto lo fu per le autorità abbaziali costrette per lo stesso motivo a trascurare i compiti più consoni alla loro carica. Nel 598, Alessandro il vir magnificus genero dello scolastico Felice nel quale ci siamo già imbattuti, de quibusdam rebus causam habere dinoscitur con la badessa del monastero di Santa Maria a Napoli Tecla. E la trascinava da tempo, promettendo ad arte di dirimerla senza poi fare seguire alle parole i fatti. Tutto era collegato all'annosa faccenda che aveva coinvolto, secondo la nostra precedente ricostruzione, Rusticiana, lo sposo Felice e Alessandro e che non si era ancora conclusa. Non fu semplice per Tecla occuparsi della lite, difendere i diritti del suo istituto contro un avversario così quotato. Al suo fianco fu posto il defensor in Sicilia Romano, la cui competenza territoriale dipendeva dai legami con l'isola di Alessandro e di Rusticiana, promotrice del monastero, ma non è difficile immaginare quanto spesso Tecla, angosciata dall'annoso conflitto, sia stata distolta dalla cura della sua congregazione ${ }^{76}$.

Principi giuridici fondamentali e noti specialmente a quanti avevano scelto di indossare l'abito monastico furono disattesi e violati, cosicché Gregorio fu spesso consultato su questioni di storno dei possedimenti monastici perpetrato attraverso uno strumento, quello testamentario, assolutamente inutilizzabile dopo la monacazione. Fra i tanti episodi che attestano il ricorso a questi atti irregolari, taluni emergono con forza. Ad accettare metà degli averi testati in punto di morte da un monaco di nome Giovanni era stato addirittura il defensor Fantino, allora probabilmente alle dipendenze del rector di Sicilia Pietro e più tardi egli stesso defensor e rector della pars palermitana ${ }^{77}$. Stranamente il papa si mostrò molto indulgente con il suo amministratore. Nonostante tutto, gli fece consegnare quanto donatogli, non mancando di ricordargli nel contempo che non era bene aspettarsi vantaggi personali dall'esercizio del proprio incarico.

Ben diverso è il piglio con il quale condannò invece il comportamento di Sirica, già badessa del monastero cagliaritano dei Santi Gavino e Lussurio, la quale, secondo il dettagliato rapporto della badessa pro tempore Gavinia, nel più evidente disprezzo della legge aveva legato ad estranei proprietà che ella, non avendole destinate altrimenti prima della monacazione, aveva obbligatoriamente dovuto trasmettere al monastero. Per sfuggire però alle norme regolari, Sirica si era avvalsa di un espediente puntualmente contestato e vanificato da Gavinia: non aveva mai voluto indossare la vestis monachica e si era servita di quella presbiteriale. Una sorta di insubordinazione che Gregorio addossava alla mancata sorveglianza del vescovo locale Gianuario. Atteso che beni di Sirica erano giunti nella disponibilità di uno xenodochio, prescriveva di incardinare un'attenta inchiesta per rintracciare la provenienza del patrimonio dell'ospizio e di procedere senza ritardi alla restituzione di tutti quei cespiti che risultassero discendere dall'atto testamentario di Sirica e non da contratti precedenti, perché, chiosava, «tutto ciò che è compiuto contro legge bisogna ritenerlo non solo inutile ma anche corrotto» ${ }^{8}$.

Gregorio invocava la legge e le procedure legali come unici strumenti legittimi e utili per dirimere qualsiasi contrasto; si affidava pertanto alla consultazione di documenti autentici e ai riscontri di indagini inappuntabili. Nel settembre del 592, infatti, scrivendo in merito alla proprietà di metà di una non meglio indicata domus all'abate del monastero siracusano di Santa Lucia, Giovanni, cui addebitava certe condotte inaccettabili sue e dei suoi monaci, chiariva: «Quanto alla metà della casa, vogliamo vedere l'atto autentico: di fronte a copie non possiamo fare nulla. Comunque, ho dato ordine al difensore Fiorentino che, se a lui consta la verità, vi restituisca tale parte. Fatta questa restituzione, l'altra metà, o ve la diamo in enfiteusi o compiamo una commutazione di redditi $\gg 79$.

Il quadro che emerge dall'epistolario gregoriano resta tuttavia sconfortante: non pochi sono gli ufficiali pubblici denunciati ai loro superiori perché restituissero il maltolto ${ }^{80}$; homines ecclesiae nostrae, che, favoriti dall'inerzia delle autorità diocesane, detenevano fondi dei cenobi e ne incameravano le rendite ${ }^{81}$; avidi abati $^{82}$ e, talvolta, familiari dei religiosi

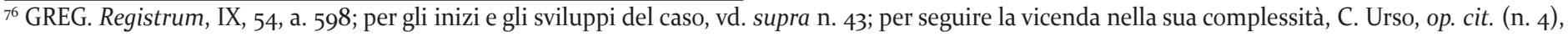
p. $87-90$.

77 GREG. Registrum, I, 42, a. 591. In un episodio, quello che riguarda l'abate Probo, dallo stesso Gregorio costretto in circostanze non note a lasciare il suo eremitaggio, a farsi monaco e ad assumere la carica di abate senza avere potuto prima disporre dei suoi beni a favore del figlio Gregorio, si consente di non rispettare la normativa vigente: XI, 15, a. 6oo; mentre, in un altro, che ha per protagonista Adeodato, monaco di Sant'Adriano a Palermo, si permette allo stesso di mettere per iscritto l'elenco dei beni - case, orti, terre e vigne - che aveva donato ai suoi schiavi prima ancora di monacarsi, perché «non compi nulla di nuovo - ciò che è vietato per legge -, ma esprimi quello che è stato fatto in passato e lo affidi per il bene alla memoria di quanto è scritto, per stroncare ogni litigio» (XIII, 3, a. 6o2).

${ }^{78}$ Su Sirica, Ibidem, IX, 198, a. 599; per le disposizioni legislative di riferimento (Novellae, 123, 38, p. 621) e per la cosiddetta legge inabilitante invocata da Gregorio (Codex Justinianus, I, 14, 5, 1, p. 68), vd. ancora utile, R. SORACI, Ius, aequitas e trasparenza amministrativa in Gregorio Magno, in L. Giordano (a cura di), Gregorio Magno. Il maestro della comunicazione spirituale e la tradizione gregoriana in Sicilia, Catania, 1991, p. 62 ss. Per le disposizioni impartite da Gregorio in casi simili, vd. anche VIII, 12, a. 598, a proposito di una lite fra Candido, abate di Sant'Andrea al Clivus Scauri di Roma, e il gloriosus maestro delle milizie Maurenzio, che si disputavano l'eredità del fratello di quest'ultimo, Giovanni, vissuto nel monastero.

${ }^{79}$ GREG. Registrum, III, 3, a. 592. Sul monachesimo d'età gregoriana a Siracusa e sui problemi affrontati in merito da Gregorio, vd. E. CALIRI, op. cit. (n. 53), p. 56-62 e passim; G. SALVO, Monachesimo e monasteri siracusani nel VI secolo, in F.P. Rizzo (a cura di), Di abitato in abitato. In itinere fra le più antiche testimonianze cristiane degli Iblei, Atti del Convegno internazionale di studi (Ragusa-Catania, 3-5 aprile 2003), Pisa-Roma, 2005, p. 117-133. Un esame più ampio è in S. BORSARI, Il monachesimo bizantino nella Sicilia e nell'Italia meridionale prenormanne, Napoli, 1963, p. 22-32; L. CRACCO RUGGINI, Il primo cristianesimo in Sicilia (III-VII secolo), in V. Messana, S. Pricoco (a cura di), Il cristianesimo in Sicilia dalle origini a Gregorio Magno, Atti del convegno di studi organizzato dall'Istituto teologico-pastorale “Mons. G. Guttadauro" (Caltanissetta, 28-29 ottobre 1985), Caltanissetta, 1987, p. 114 ss. ${ }^{80}$ GREG. Registrum, I, 46: sull'ufficiale Donato che si era appropriato di un bene appartenente al monastero sardo di San Vito e che doveva essere costretto dal dux Sardiniae Teodoro, della cui protezione forse godeva, a comparire in giudizio.

${ }^{81}$ Ibidem, II, 26, a. 592: sul presbitero della massa «detta di Marotide» che pretendeva di incamerare parte delle entrate del monastero siciliano di San Pietro, dovute alla generosità dei fedeli, invocando una traditio; II, 50, a. 592: sulle monache del monastero ubicato nel fondo Monosteo in Sicilia che si erano viste sottrarre ab ecclesia nostra il fondo Villa-nova ricevuto in eredità, assieme alle relative pensiones di due indizioni; VII, 40, a. 597: sulla situazione creata dall'arroganza di alcuni chierici ravennati (V, 1, a. 594; VI, 28, a. 596; su cui supra e infra, n. 20, 89), i quali «ritengono [i monasteri] come delle
} 
e altri "laici", che attentavano al patrimonio monastico e si intromettevano nella sua gestione per avvantaggiarsene ${ }^{83}$. Talvolta in questo malaffare erano implicati i più alti livelli dell'ordo, se è vero che lo stesso rettore di Sicilia Pietro aveva "tardato" a trasferire ad alcuni monasteri i lasciti del defensor Antonino ${ }^{84}$, e che Gregorio, avendo avuto con ogni probabilità sentore di azioni illecite messe in atto dal vescovo di Napoli Fortunato, si fosse sentito in dovere di "avvertirlo" perché non si impossessasse dei donativi devoluti agli enti guidati dalla badessa Graziosa e dall'abate Teodosio ${ }^{85}$. Anche la condotta di quei vescovi che, subito dopo la morte di un abate, approfittavano del momento di vacanza della carica abbaziale per intromettersi nell'inventariazione dei possedimenti del cenobio, destava sospetti ${ }^{86}$. Non a caso Gregorio, fra i privilegi concessi nel 598 al monastero dei Santi Giovanni e Stefano in Classe, aggiungeva che «l'inventario dei beni e delle carte [...] non sia compiuto dagli ecclesiastici, ma tale inventario sia fatto, quando è necessario, dall'abate del luogo con gli altri abati» ${ }^{87}$. E che dire degli ordinari diocesani che dai monaci si facevano rimettere quaedam de monasterio sub exenii quasi specie, ovvero sotto forma di doni offerti dai coloni ai padroni ${ }^{88}$ ? Oppure di quelli che, citando in maniera subdola le norme legislative che impedivano ad un vescovo di testare possedimenti della Chiesa, tentavano di contestare ai monasteri la proprietà di beni provenienti invero da atti testamentari vescovili, ma in virtù di procedure già validate dall'autorità pontificia ${ }^{89}$ ?

La casistica si amplia se recuperiamo nel Registrum i dati relativi all'amministrazione degli xenodochia, ospizi che erano per lo più collegati ad un istituto monastico spesso per volontà testamentaria di pii benefattori e rappresentavano anch'essi una testimonianza concreta della presenza della Chiesa nelle città e nel territorio ${ }^{90}$. Tanto più che talvolta erano ubicati lungo strade di comunicazione importanti e molto frequentate. Quello di Via Nova a Roma, ad esempio, si trovava «in prossimità della porta Appia [...], lungo la direzione che percorrevano i pellegrini [...] che si recavano a visitare i santuari della via Appia-Ardeatina» ${ }^{91}$.

Questi impianti, cui si riconosceva una profonda valenza sociale, erano per ciò stesso gratificati dall'appoggio della curia pontificia e da più o meno consistenti legati testamentari. Alcuni esempi possono bastare. Uno xenodochium era sicuramente annesso al monastero di San Teodoro nel palermitano: conosciamo i nomi del fondatore, Pietro, e di un direttore, Bacauda, già morto tuttavia nel 590, e sappiamo che era ben dotato di mezzi finanziari. Nell'ottobre del 598, infatti, si consigliava al defensor palermitano Fantino di associare alla suddetta fondazione il terreno e le altre donazioni destinate da Isidoro, illustris memoriae, all'edificazione di un altro ospizio sempre nella città di Palermo, se quest'ultimo non avesse corredato il suo progetto di fondi sufficienti. Fatto che peraltro avrebbe spiegato perché gli eredi chiamati a realizzarlo avessero eluso l'obbligo, rischiando di incorrere nei rigori della legge ${ }^{92}$. Per sostenere, inoltre, l'azione di un tale abate Giovanni del monte Sinai che si stava occupando di completare una struttura d'accoglienza riservata agli anziani, uno hierochomius ${ }^{33}$ (un unicum nell'Epistolario!) istituito da un tale Isauro, da Roma si prometteva l'arrivo di

proprietà», che indusse Gregorio a intimare loro di non entrare in un monastero se non per pregare o celebrare la Messa; IX, 88, a. 599: sulla lamentela di Stefano, abate del monastero di San Marco a Spoleto, relativa ad un fondo sito nel territorio di Minturno in Campania, già donato da papa Benedetto I al suddetto istituto, indebitamente trattenuto da uomini di Chiesa o, forse, ipotizza Gregorio, da estranei; IX, 205, a. 599: sul chierico che si era impadronito di alcuni averi che una vedova, della quale egli stesso curava il patrimonio, aveva testato al monastero cagliaritano di San Giuliano.

${ }^{82}$ Del settembre 602 è la lettera inviata al vescovo di Cagliari, in cui si denuncia lo spregiudicato comportamento dell'abate Giovanni, che si rifiutava di restituire alla badessa Desideria «i beni dei suoi genitori e anche di suo fratello, spettanti regolarmente a lei [...] e detenuti in modo indebito dall'abate»: Ibidem, XIII, 4, a. 602.

${ }^{83}$ La consuocera della religiosa sarda Pompeiana, ad esempio, tramava affinché fosse annullato il testamento del proprio figlio che aveva destinato parte dei suoi averi alla realizzazione di una struttura monastica: Ibidem, I, 46, a. 591, e supra, n. 38; Fidenzia, madre della monaca Adeodata, è accusata di "defraudare" la figlia: IX, 87, a. 599. Proprio per evitare il ripetersi di attacchi ai beni delle comunità ad opera di laici, certo meno controllabili dalle autorità religiose, Gregorio aveva richiesto ai vescovi di assegnare i compiti gestionali solo a chierici. L'indicazione però era spesso disattesa: Ibidem, IX, 204, a. 599, al defensor sardo Vitale, cui riferisce della trascuratezza del vescovo di Cagliari Gianuario, imputato in IX, 205 proprio di consentire che a laici fosse affidato il patrimonio della chiesa cagliaritana; e, sullo stesso tema, XIV, 2, a. 603, in cui Gregorio invita il rettore sardo Vitale a consegnare la cura dei patrimoni di ospizi e altri istituti religiosi all'economo della chiesa di Cagliari e all'arciprete Epifanio.

${ }^{84}$ Ibidem, II, 50, a. 592.

${ }^{85}$ Ibidem, III, 58, a. 593; V, 50, a. 595.

${ }^{86} \mathrm{Ibidem}, \mathrm{V}, 49$, a. 595: in questo caso l'ammonizione di Gregorio è rivolta al vescovo di Rimini, Castorio, e riguarda il monastero dei Santi Andrea e Tommaso, su cui vd. anche $V$, 47, a. 595.

${ }^{87}$ Ibidem, VIII, 17, a. 598.

${ }^{88}$ Ibidem, VIII, 32, a. 598: l'abate del monastero del "Vivario o di Monte Castello" aveva consegnato donativi diversi e ceduto al vescovo di Squillace donationis titulo anche un terreno per edificarvi una chiesa. Gregorio ordina che, dopo aver calcolato il terreno effettivamente necessario allo scopo, il resto sia immediatamente restituito alla legittima proprietà. Per il significato di exenium, GREG. Lettere/3, p. 88, n. 5; per il contesto calabro, S. BETTOCCHI, La Calabria nel Registrum Epistularum di Gregorio Magno, in Vetera christianorum, 35, 1998, p. 17-38.

${ }^{89}$ GREG. Registrum, VI, 1, a. 595: il vescovo in questione è Giovanni, predecessore di Mariniano, le cui disposizioni testamentarie erano da considerarsi nulle, con l'eccezione di quelle relative al monastero di San Apollinare in Classe dedicato ai santi Marco, Marcellino e Felicola (GREG. Lettere/2, p. 273, n. 4), perché confermavano solamente trasferimenti di beni personali decisi in vita e comunicati all'autorità pontificia che li aveva approvati. Dall'epistola VI, 28 del marzo 596, sei mesi dopo quella appena commentata, si apprende che Mariniano, forse indotto in errore da cattivi consiglieri che pare peraltro avessero esteso la loro colpevole cupidigia ad altri istituti, era intenzionato ad agire diversamente, intaccando gli interessi del monastero ravennate. Tre anni dopo, Gregorio ritorna sulla questione, affidandone la valutazione al notaio Castorio (IX, 169, a. 599).

${ }^{90}$ Sul tema specialmente H.W. DEY, Diaconiae, xenodochia, hospitalia and monasteries: 'social security' and the meaning of monasticism in early medieval Rome, in Early Medieval Europe, 16, 4, 2008, p. 398-422; cfr. anche F.R. STASOLLA, A proposito delle strutture assistenziali ecclesiastiche: gli xenodochi, in Archivio della Società romana di Storia patria, 121, 1998, p. 5-45; A.M. GIUNTELLA, op. cit. (n.15), p. 88 ss.; F.R. STASOLLA, op. cit. (n. 15), p. 223 ss. ${ }^{91}$ GREG. Registrum, I, 42, a. 591; la citazione nel testo è di A.M. GIUNTELLA, op. cit. (n. 15), p. 101.

${ }_{92}$ Su questo ospizio: GREG. Registrum, I, 9, a. 590; IX, 35, a. 598; IX, 171, a. 599.

${ }_{93}$ Sul termine, DU CANGE, Glossarium mediae et infimae latinitatis, IV, rist. Graz 1954, v. Hierocomium = Gerocomium, p. 6o: Locus ubi aluntur senes. 
«quindici coperte, trenta mantelli di lana rozza e quindici letti; [...] anche il denaro per comprare coltri e pagare il noleggio della nave» ${ }^{94}$.

Il numerarius ${ }^{95}$ Bonifacio lasciò una parte delle sue proprietà ad un ospizio posto presso la basilica di San Pietro a Roma, probabilmente lo xenodochium S. Gregorii iuxta gradus $S$. Petri $i^{96}$. I cenni sulla necessità di presentare, tramite il vir clarissimus Lorenzo, a sua volta aiutato dallo scribo Azimarco nonché dal rector Romano e dal vescovo di Siracusa Giovanni, i rendiconti di Bonifacio presso l'ufficiale competente - ovvero, l'ex console Leonzio -, salvaguardando nel contempo gli interessi della moglie affidata a Gregorio dallo stesso contabile, fanno supporre una situazione non chiara. Infatti, passati dei mesi dalla data della prima lettera del novembre-dicembre 598, il pontefice si rivolgeva direttamente a Romano per consigliargli di consegnare a Leonzio l'inventario degli averi di Bonifacio attestante lo stato di povertà in cui questi era morto, e per proporre all'ex console una transazione a conclusione dell'intera faccenda. Nell'eventualità che la proposta non fosse stata accettata, Gregorio si diceva pronto a restituire la donazione devoluta all'ospizio romano piuttosto che sopportare inanis invidia ${ }^{97}$. Anche a Gerusalemme era accaduto qualcosa di insolito a proposito dell'utilizzo dei solidi che un tale Probo aveva elargito per costruire uno xenodochio. Il pontefice, in questa occasione, si disse però impossibilitato a cambiare quod deliberatum fuerat e inviò 50 solidi a Filippo, il presbitero che aveva presentato la denuncia ${ }^{8}$.

Per chiudere, infine, lo scontro sulla titolarità dei fondi palermitani di Faiano, Nasoniano e Libiniano che da tempo contrapponeva i predecessori di Domizio, abate del monastero palermitano dei Santi Massimo e Agata, ovvero Lucuscano, e del suddiacono Antonio, praepositus dell'ospizio Valerio di Roma, Gregorio li invitava a sottoscrivere la soluzione che era stata individuata, ma non esplicitata per la verità nella lettera, e si faceva garante del patto e della nullità di ogni ulteriore atto volto a riprendere la lite99.

Appare evidente come egli fosse propenso a comporre nel più breve tempo possibile ogni tipo di controversia e come in questo senso indirizzasse i suoi interlocutori, convinto che «è degno di lode e consono allo zelo religioso preferire la pace gradita a Dio ai guadagni materiali e scambiare con i beni che passano i profitti della carità che permangono per sempre». Né questo auspicio né le continue raccomandazioni su una corretta e limpida amministrazione erano però sempre onorati dai suoi homines che, secondo l'accusa rivolta al defensor Romano, pensavano magis utilitatibus quam mercedibus ${ }^{100}$, oppure - e in questo si era distinto il vescovo di Cagliari Gianuario - non si affrettavano a visionare i rendiconti che i responsabili erano tenuti a depositare ogni anno ${ }^{101}$.

L'oculata gestione era invece fondamentale anche per scongiurare che nei monasteri le condizioni di vita, segnate pesantemente dal contesto politico e militare, peggiorassero fino a trascinare tutti nella miseria più nera. In quei decenni infatti, verosimilmente per cause da addebitare anche al cattivo o incompetente governo dei patrimoni, o magari al calo drastico delle rendite fondiarie, oppure a ruberie e sottrazioni di ogni genere, avveniva sempre più spesso che Roma, allarmata dalle notizie che provenivano da più parti, fosse costretta ad organizzare azioni di soccorso.

Informato che le ancillae Dei quaedam Nolanae civitatis in Aboridana domo commanentes non disponevano sufficientemente neanche di vitto e di vestiti, Gregorio chiedeva al rector campano, Antemio, di provvedere con una dazione iniziale di 40 solidi d'oro, seguita da una rendita annua di 20 solidi $^{102}$. E rassicurava sul suo sostegno i monaci calabresi del monastero di Sant'Angelo a Tropea, che lamentavano anch'essi victus necessitas, a patto che si potesse dimostrare la loro rettitudine di vita. In tale evenienza sarebbero stati sgravati anche dal fitto di 1 solido e 2 tremissi dovuto per un appezzamento di terra della Chiesa, che sarebbe stato concesso libellario nomine, a titolo di livello cioè, e per la cifra simbolica di solo un tremisse ${ }^{103}$.

Su pochi mezzi potevano contare il monastero siciliano di San Giorgio nella tenuta di Marotide ${ }^{104}$ e forse anche quello agrigentino di Santo Stefano presieduto dalla badessa Domina ${ }^{105}$. Sempre in Sicilia, 10 solidi furono assegnati al monastero eretto a Catania dal vir gloriosus Giuliano, che si era mostrato restio a segnalare la situazione e fu per questo affettuosamente ripreso dal pontefice ${ }^{106}$. In Africa, la nume-

94 GREG. Registrum, XI, 2, a. 600.

${ }_{95}$ Sulla carica di "contabile", GREG. Lettere/3, p. 197, n. 2, anche per i riferimenti legislativi.

${ }^{96}$ Ibidem, p. 197, n. 3.

${ }^{97}$ GREG. Registrum, IX, 63, a. 598; IX, 131, a. 599.

${ }_{98}^{8}$ Ibidem, XIII, 26, a. 603; sul presbitero non si hanno altre notizie.

99 Ibidem, IX, 67, a. 598; la conferma di quanto stabilito è in IX, 83 dello stesso anno.

${ }^{100}$ Ibidem, II, 50, a. 592.

${ }^{101}$ Ibidem, IV, 24, a. 594.

${ }^{102}$ Ibidem, I, 23, a. 591; nella stessa lettera si disponeva la consegna di 2 solidi ciascuno, ma una tantum, al presbitero e ai due monaci dell'oratorio di San Michele Arcangelo, nel Castro Lucullano. Sugli interventi pontifici, volti a soccorrere tutti gli indigenti e non solo monaci e monache, che debbono essere catalogati, se non proprio come "elemosine" (così invece G. JENAL, op. cit. [n. 2], p. 225, 245), forse solo come fatti eccezionali e non come pratiche istituzionalizzate (P. DELOGU, Le passage de l'Antiquité au Moyen Âge, in A. Vauchez [dir.], Rome au Moyen Âge, nouv. éd., Paris, 2010, p. 49), ma che consentirono comunque al pontefice di assumere il ruolo di protettore della popolazione romana in quei tragici decenni (H.W. DEY, op. cit. [n. 9o], p. 399), vd. fra i tanti M. FORLIN PATRUCCO, La vie quotidienne dans la correspondance de Grégoire le Grand, in J. Fontaine, R. Gillet, S. Pellistrandi (éd.), Grégorie le Grand, Colloque Internationaux du Centre National de la Recherche Scientifique (Chantilly, 15-19 septembre 1982), Paris, 1986, p. 59-68; e, più di recente, O. BUCARELLI, op. cit. (n. 40), p. 421-436.

${ }^{103}$ GREG. Registrum, II, 1, a. 591. Sull'enfiteusi ecclesiastica e sulla locazione più in generale, che non potevano superare i trent'anni: Novellae, 7, 3; 120, 3, p. 54-56, 580 .

${ }^{104}$ GREG. Registrum, II, 26, a. 592.

${ }^{105}$ Ibidem, VIII, 23, a. 598.

${ }^{106} \mathrm{Ibidem}$, XIII, 21, a. 603. Sulla partecipazione attiva dei nobiles nelle azioni pontificiea sostegno degli indigenti, vd. R. RIZZO, Il coinvolgimento dei nobili ad bonum dispensandum: teoria e pratica del bene in Gregorio Magno, in L. Giordano, M. Marin (a cura di), Gregorio Magno, un letterato al governo, Convegno di studi dedicato a don Vincenzo Recchia (Catania, 1-2 dicembre 2011) = Auctores nostri, 11, 2012, p. 193-224, specialmente p. 193-194. Ritengo tuttavia che i 
rosissima comunità che viveva sotto la guida dell'abbadessa Adeodata doveva mancare di tutto, se da Roma si allestiva l'invio in loco di una nave colma di ogni bene. La spedizione fu affidata ad un tale Vitale, latore di una lettera pontificia al rettore d'Africa Ilaro, delegato ad attendere l'arrivo della nave e ad evitare che sul suo carico pesassero onera e angariae ${ }^{107}$.

A Roma, lo stato miserevole delle congregazioni monastiche era stato aggravato in quegli anni dalle continue incursioni dei Longobardi. In una lettera del marzo 595 all'imperatore Maurizio, Gregorio descriveva con parole accorate i pesanti disagi che affliggevano molte delle monache giunte, profughe, nella città per sottrarsi alla captivitas dei nemici. Non era stato possibile alloggiarle tutte nei monasteri femminili romani e molte vivevano da sole in estrema miseria. Il papa aveva distribuito anche a loro «quanto avanzato ai ciechi, ai mutilati, agli altri invalidi» delle 30 libbre d'oro ricevute da Maurizio ${ }^{108}$. Due anni dopo, nulla era cambiato. Anzi, le monastriae - come quelle erano chiamate in greco - erano diventate numerosissime: iuxta notitiam [elenco] qua dispensantur tria milia repperiuntur. La Chiesa romana, dunque, a quella data aveva in carico ben tre mila monache, molte delle quali erano delle rifugiate. Ogni anno per far fronte alle loro esigenze erano prelevate dal tesoro di San Pietro 8o libbre, vale a dire circa 5760 solidi, ma quelle povere donne erano troppe e a Roma in quel tempo i prezzi erano lievitati in maniera esponenziale. Non sempre si era in grado di risolvere i loro bisogni. In questo quadro dovette sembrare provvidenziale l'ulteriore rimessa, nel 597, da parte questa volta della patrizia Teoctista, sorella dell'imperatore, di altre 30 libbre d'oro per il riscatto dei prigionieri e, più in generale, per soccorrere i poveri. Metà dell'intero importo, non un "resto" più misero come era stato deciso nella precedente distribuzione, la curia decise che fosse impiegata per acquistare coperte con le quali quelle derelitte avrebbero potuto affrontare meglio i rigori dell'inverno ${ }^{109}$.

La generosità di Gregorio talvolta era veramente sorprendente: apprezzando il senso di moderazione e di rispetto dimostrato dall'abate provinciae Isauriae Elia, non solo accolse la sua richiesta d'aiuto ma addirittura incrementò, con una strepitosa rispondenza di rinunce da una parte e di altrettanti aumenti dall'altra, l'importo finale del contributo. «Avete voluto che vi fossero inviati cinquanta solidi d'oro per le necessità del monastero. Pensando, poi, che fossero troppi, me ne avete condonati dieci, così che ve ne mandas- simo quaranta. Ma affinché forse, ciò non fosse oneroso, avete giudicato bene condonarmene altri ancora. Ma poiché vi abbiamo trovato molto benevolo nella moderazione verso di noi, alla medesima benignità rispondiamo con un ricambio simile. Vi abbiamo mandato cinquanta solidi e, se mai - forse - fosse poco, ne abbiamo aggiunti altri dieci. Se mai in realtà, anche questo fosse pur sempre poco, ne abbiamo fatti aggiungere altri dodici ${ }^{110}$. Per un totale di 72 soldi, ovvero 1 libbra d'oro.

Sempreallo scopo di favorire la sopravvivenza dei religiosi, bastava in certi contesti procurare loro terre da coltivare. La dazione al monastero di Blera di due campi, da possedere salvo iure ecclesiae nostrae in triginta annorum spatium, commissionata al notaio Eugenio, rettore della Tuscia, mirava proprio a rendere autosufficiente la comunità' ${ }^{11}$. Allo stesso fine, il defensor campano Antonino aveva testato ad monastero pecunia [...] ad emendas possessiones. Per eseguire le volontà del defunto benefattore, Gregorio aveva mandato a Sorrento alcuni monaci, i quali invece di impegnare proficuamente il danaro lo stavano dilapidando. C'era il serio rischio che esaurissero le risorse e che non potessero neanche procacciarsi il vitto giornaliero con il prodotto dei terreni che tardavano a comprare. Solo l'azione tempestiva del nuovo defensor, Antemio, poteva impedire quello spreco ${ }^{112}$.

\section{I MONASTERI: LUOGHI DI DISORDINI E DI PENITENZA}

Nei monasteri comunque regnava più che altrove una certa vivacità d'azione e di pensiero, certo collegata ad un altrettanto dinamico humus culturale, che si riscontra anche presso le congregazioni femminili. Colpisce, a questo proposito, che in molti casi denunce di gravi accadimenti, di molestie, di attacchi all'ordine e al quieto vivere delle sanctimoniales fossero fatte giungere a Roma direttamente dalle badesse e dalle religiosae feminae. Alcune avevano vergato probabilmente di propria mano le missive a significare che godevano di una buona preparazione culturale, conseguita ancora prima di monacarsi o proprio in vista di quella scelta, oppure acquisita o quantomeno affinata nei chiostri, che, recuperando le norme regolari già esistenti dai tempi di Cesario d'Arles e dello stesso Benedetto, possiamo immaginare come luoghi consoni e inclini a queste attività"13. Non per questo tuttavia quegli ambienti erano esenti da intrighi e violenze.

10 soldi a cadenza annuale che Gregorio stanziò a vantaggio dell'istituto catanese fossero diretti, in questo contesto, non «a far fronte ai bisogni dei poveri all'interno del [...] monastero», come scrive la studiosa, bensì ai problemi economici dell'intera comunità monastica. Non a pauperes in senso generico e generale, dunque, ma al monastero erano destinate res pauperum che lo stesso Giuliano era invitato a ricevere sine iniuria, considerandole un dono di Pietro. Diversa la situazione del monastero siracusano di Santa Lucia, per il quale il riferimento all'hospitalitas riservata ai poveri è esplicito: GREG., Registrum, III, 3, a. 592. Sulla fondazione catanese, vd. B. SAITTA, Catania nel Registrum epistolarum, in ID., Catania nel Medioevo, II ed., Catania, 2008 , p. 15.

${ }^{107}$ GREG. Registrum, IX, 133, a. 599.

${ }^{108}$ Ibidem, V, 30, a. 595.

${ }^{109}$ Ibidem, VII, 23, a. 597. Può essere interessante riflettere sul rapporto fra la popolazione monastica e quella laica nel periodo del pontificato di Gregorio, allorquando, secondo L. GATTO (Riflettendo sulla consistenza demografica della Roma altomedievale, in Roma medievale. Aggiornamenti, op. cit. [n. 53], p. 152), «i romani scenderanno anche al di sotto dei 100 mila»; di parere diverso P. DELOGU (op. cit. [n. 102], p. 43 e $39-88$ sul tema; cfr. G. JENAL, Gregor der Große und die Stadt Rom, in F. Prinz [herausg.], Herrschaft und Kirche, Beiträge zur Entstehung und Wirkungsweise episkopaler und monastischer Organisationsformen, Stuttgart, 1988, p. 109-145), per il quale si trattava ormai «di decine e non più di centinaia di migliaia di abitanti»; sull'evidente capacità della città «di garantire luoghi di accoglienza sufficientemente ampi», invece, vd. L. ERMINI PANI, op. cit. (n. 66), p. 19-47, in particolare p. 44-45.

${ }^{110}$ GREG. Registrum, V, 35, a. 595.

"in Ibidem, IX, 97, a. 599.

${ }^{112}$ Ibidem, IX, 106, a. 599

"13 In GREG. Registrum, III, 58, a. 593, è la badessa Graziosa a inviare una sua petizione fino a Roma. Vd. sul punto, S.F. WEMPLE, Female monasticism in Italy and its comparison with France and Germany from the ninth through the eleventh century, in W. Affeldt, U. Vorwerk (herausg.), Frauen in Spätantike und Frühmittelalter. Lebensbedigungen - Lebensnormen - Lebensformen. Beiträge zu einer internationalen Tagung am Fachbereich Geschichtswissenschaften der Freien Universität Berlin, Sigmaringen, 1990, p. 293; EAD., op. cit. (n. 4o), p. 79; C. URSO, op. cit. (n. 4), p. 83. 
Al contrario. Molte religiose feminae, fra le quali ci limitiamo a ricordare la sarda Pompeiana, lamentavano di subire molestie varie ${ }^{144}$, ma la circostanza più clamorosa ed inquietante al tempo stesso fu portata a conoscenza del pontefice nel 599 dalla badessa Agnella nel cui monastero di Napoli da tempo erano alloggiati dei soldati, mentre il vescovo diocesano Fortunato sembrava non avvertire l'urgenza di allontanarli, in maniera che «l'astuto nemico [...] non possa gioire dell'inganno teso alla vita religiosa $»^{115}$. Anni dopo, nonostante le ferme prese di posizioni di Gregorio, sempre a Napoli e sempre a causa della presenza nel territorio di una «milizia senza freno», un soldato aveva sedotto una monaca e il suo superiore, il duca Guduin, non si era neanche preoccupato di punire il colpevole in maniera esemplare $e^{116}$.

Un altro fronte era rappresentato dalle continue aggressioni che minacciavano, come si è sottolineato, i patrimoni monastici e costringevano abati e badesse a vigilare sui contenziosi che ne scaturivano. Nonostante che queste causae di norma fossero rimandate dal pontefice alle decisioni di giudici elettivi, rimanevano spesso di non semplice soluzione e soprattutto annose. Potevano indurre le autorità monastiche a trascurare i loro compiti e, di conseguenza, diventare deflagranti per il sistema stesso. Appunto per scongiurare questo pericolo e sgravare Giovanni, abate del monastero siracusano di Santa Lucia, di tutte le pratiche che appesantivano il suo ufficio, si suggerì da Roma che il suddiacono Pietro le trasferisse all'attenzione di Fausto, già cancelliere dell'ex pretore Romano ${ }^{117}$. Il contenuto di una lettera, successiva a questa dell'agosto 591, attesta che Fausto probabilmente non accettò l'incarico e che l'abate Giovanni rimase oberato dai tanti negotia. E, da quanto si legge nella missiva, c'è da pensare che questi non fu sempre all'altezza della situazione. Il monastero, a motivo della sua neglegentia, o è forse meglio dire della sua imperizia, aveva visto compromesso il suo prestigio, tanto da richiedere decisioni drastiche. Tu, scriveva Gregorio a Giovanni, «non andrai frequentemente fuori del monastero. Per queste vicende nomina un procuratore, e tu attendi alla lettura e alla preghiera $»^{118}$.

Tali incombenze, ovviamente, risultavano ancora più cariche di pericolosi esiti, se a doversene curare erano monache e badesse. A Napoli, fu per esempio la badessa Flora ad essere quasi sopraffatta da una congiuntura dai contorni non sempre nitidi; in Sardegna, per l'indolenza del vescovo di Cagliari, «donne di per sé dedite al servizio di Dio, sono gravate, tramite persone pubbliche con tributi e altri servizi. Esse sono inoltre obbligate, per sopperire alle pretese del fisco, ad andare per villaggi e poderi, e a mischiarsi senza competenza in faccende proprie degli uomini. La fraternità tua rimuova prontamente questo inconveniente». Quello che paventava Gregorio, d'altronde, pare fosse già avvenuto, visto che qualcuna ad lapsus [...] deducta est ${ }^{119}$. Solo se libere da altre occupazioni e superata ogni costrizione, ebbe a dire anni dopo, le monache - in questo caso erano quelle di un istituto sottoposto alla giurisdizione del vescovo di Luni «potranno con mente tranquilla perseverare assiduamente nella preghiera e nelle lodi di Dio». Potranno cioè avviarsi verso la salvezza e verso Dio, vivendo santamente nel luogo in cui meglio che altrove quel cammino era realizzabile ${ }^{120}$.

Nell'eventualità contraria, ove cioè fossero prevalse le apprensioni mondane, la possibilità di cadere nel peccato o di assumere atteggiamenti sempre più irrispettosi dei precetti fondamentali della scelta monacale si faceva sempre più concreta. Lo certificano le cospicue notizie che giungevano a Roma su religiosi e religiose che ritornavano nel secolo spogliandosi dell'habitus monastico, o che, nel chiostro, commettevano reati di ogni tipo.

Per quanto attiene al primo punto, il riscontro più noto riguarda un antico amico del pontefice, il patrizio Venanzio, il quale aveva lasciato il chiostro, si era sposato con Italica e ne aveva avuto due figlie, Antonina e Barbara. Delle vicissitudini personali di Venanzio e delle sue "donne", a noi preme in questa sede solo ricordare la rinuncia del patrizio all'abito monastico e le tante inutili esortazioni di Gregorio all'amico perché recedesse dalla sua colpevole decisione. Colpevole è Venanzio più ancora che se avesse rubato denaro, visto che aveva sottratto a Dio se stesso dopo essersi a Lui votato ${ }^{121}$.

Certo le spinte verso l'abbandono del chiostro si sostanziano di motivi che spaziano da una vocazione non sufficientemente "comprovata" dai superiori - fu questa verosimilmente la causa della fuga dal monastero retto dall'abate Barbaziano di un certo Maurizio, precocemente tonsurato, e di altri che ne avevano imitato l'esempio ${ }^{122}-$, al prevalere degli appetiti sessuali, o addirittura alla decisione di rifugiarsi presso i nemici longobardi, nella speranza di scampare alla ferocia delle loro incursioni. Questa decisione pare avesse adottato nel 600 un monaco del monastero di Sant'Arcangelo, sito forse a Napoli o comunque in territorio campano, il cui abate, accusato di connivenza, era stato pesantemente intimorito dal dux Campaniae Gudiscalco. Il povero abate si era tanto spaventato da non volere più uscire dal sacro recinto. Emerge però il sospetto che il dux avesse usato quell'espediente per giustificare l'irruzione nel monastero e per saccheggiarlo. Un atto ingiustificabile. Tanto più che in quei tempi «gli schiavi di molti nobili, i chierici di molte chiese, i monaci di molti monasteri, gli uomini di molti funzionari spesso sono passati ai nemici», ma non per questo - è il monito del pontefice - si poteva addossare la colpa ai loro superiori ${ }^{123}$.

\footnotetext{
${ }^{14}$ GREG. Registrum, I, 61, a. 591; in tribunale erano finite anche le liti che angustiavano un'altra pia donna, Catella (I, 6o. 62, a. 591).

${ }^{115}$ Ibidem, IX, 208, a. 599.

${ }^{116}$ Ibidem, XIV, 10, a. 603: è al dux della citta, Guduin, che Gregorio affida la severa punizione del miles.

${ }^{117} \mathrm{Ibidem}$ I, 67, a. 591, Fausto avrebbe ricevuto per il suo impegno una seppur piccola ricompensa.

${ }^{118}$ Ibidem, III, 3, a. 592.

${ }^{119}$ Ibidem, IV, 9, a. 593. Per la vicenda che dovette affrontare Flora, vd. supra e n. 76.

${ }^{120}$ Ibidem, IX, 115, a. 599. Cfr. R. A. MARKUS, op. cit. (n. 1), p. 70-72.

${ }^{121}$ La vicenda di Venanzio si può seguire in GREG. Registrum, I, 33; VI, 42-43; IX, 13. 232; XI, 18. 19. 23. 25; XIII, 12. Le lettere coprono un arco di tempo che va dal marzo 591 al novembre 602; per l'intera vicenda, cfr. C. URSO, op. cit. (n. 4), p. 131-137.

${ }^{122}$ GREG. Registrum, X, 9, a. 6oo: Gregorio, dopo avere avanzato dubbi sulla vocazione dello stesso Barbaziano, precisa che non si doveva procedere alla tonsura prima del biennio, che serviva proprio per meglio verificare la vocazione dei nuovi religiosi.

${ }_{123}$ Ibidem, X, 5, a. 600.
} 
Anche per spiegare l'allontanamento, risalente a parecchi anni prima, dei monaci dipendenti dal vescovo Paolino di Taureana in Calabria che il suddiacono Pietro fu incaricato di rintracciare e di riunire nella sede monastica di San Teodoro in Messina, era possibile invocare impellenze collegate all'espansionismo longobardo nel Meridione. Magari, i religiosi erano stati indotti a fuggire dalla loro sede dalla violenza nemica e non si erano curati di avvisare del loro arrivo in Sicilia: si rendeva necessario a quel punto "raccoglierli" ad uno ad uno e rinchiuderli nel monastero messinese ${ }^{124}$.

Per ciò che attiene alle congregazioni femminili, gravi episodi erano avvenuti in Sardegna nel 594. Il pontefice ne aveva discusso a Roma con il presbitero della chiesa cagliaritana Epifanio, che, alla data della lettera, tornava nell'isola libero da ogni accusa precedentemente prodotta contro di lui e incaricato di fare rapporto all'autorità diocesana sui provvedimenti adottati. Non conosciamo nei dettagli quanto fu deciso nei confronti delle religiose che si erano spogliate dell'abito monastico, ma è detto che ai complici sarebbe stata inflitta la scomunica, revocabile dal vescovo solo dopo averne appurato il pentimento ${ }^{125}$. Nello stesso periodo, a Ravenna, delle monache, sicure di godere della protezione dell'esarca Romano, avevano deposto l'habitus e si erano sposate. Anche Gregorio appare scosso, incredulo e impotente davanti all'inaudita accusa contro un così importante rappresentante dell'impero ${ }^{126}$. Qualche anno dopo, in Puglia, la figlia del defunto maestro delle milizie Tulliano fu rinchiusa in un monastero sotto stretta custodia per scontare la sua colpa; ella, infatti, non aveva esitato ad allontanarsi dall'istituto che l'aveva ospitata e, proiectis religiosis vestibus, a rientrare nel secolo. La condotta immorale della monaca fu imputata al vescovo di Siponto, Vitaliano, e al defensor di Puglia e Calabria, Sergio, che avrebbero dovuto vigilare e, nimia desidia ac torpore, avevano tralasciato i loro obblighi ${ }^{127}$.

Diversa era, e come tale fu valutata, la vicenda della moglie del cartarius Leone, la quale, convinta del tradimento del marito, lo aveva lasciato e si era monacata: passati tre anni, avendo appreso della sua innocenza, era ritornata al suo fianco. Il vescovo di Taormina l'aveva privata della comunione, coinvolgendo nella severa punizione anche la sua servitù. Gregorio lo invitò a riammettere immediatamente nella comunità dei fedeli i servi, sui quali non era giusto che ricadesse la colpa della padrona, e a ridurre i tempi della privazione inflitta a quest'ultima ${ }^{128}$. Altre vicende sui generis si segnalano in Gallia, laddove la monaca Siagria era stata strappata a forza dal chiostro e costretta a sposarsi, sempre a causa dell'ignavia dei vescovi locali, Virgilio di Arles e Siagrio di Autun ${ }^{129}$; oppure a Cagliari, dove il rapitore di un'altra monaca, cognata del vir clarissimus Stefano, era un tale Pietro, il quale ab homine filii nostri magnifici Filoxeni dicitur defensari e, forte di questo appoggio, si era convinto di potere sfidare impunemente le autorità diocesane ${ }^{130}$.

In questo contesto, può essere interessante rilevare che la frequenza di eventi riconducibili ad atteggiamenti contrari alla conservazione dello status monastico produsse una vera e propria casistica di miracoli, riferiti ad episodi nei quali con l'aiuto divino si sventavano per tempo i propositi di fuga o si ritrovavano quanti invece avevano messo in atto il loro malvagio disegno. Ne racconta alcuni lo stesso Gregorio in una lettera alla patrizia Rusticiana del febbraio del $601^{131}$.

Sempre più numerosi furono anche i monachi girovagi, che conservavano l'habitus ma, contravvenendo al principio della stabilitas loci, si allontanavano dalla loro struttura per spostarsi in zone distanti dove talvolta, pur essendo colpiti da scomunica, erano accolti dagli ordinari locali. Era accaduto a Spoleto, a quanto testimoniava il presbitero e abate Valentino ${ }^{132}$, e anche in Africana provincia a vantaggio dei monaci girovaghi di un istituto guidato dall'abate Cumquodeus e sottoposto alla giurisdizione del vescovo Domenico di Cartagine ${ }^{133}$. Tre monaci del monastero napoletano di San Martino, giunti in Sicilia per sbrigare una faccenda loro affidata dall'abate Teodosio, avevano sfruttato l'occasione ed erano rimasti nell'isola ${ }^{134}$.

Naturalmente tutti costoro non osservavano più le antiche regole, né tanto meno le disposizioni disciplinari: nella diocesi sorrentina, in Campania, molti non si fecero scrupolo di usare per le proprie necessità danaro personale. Un fatto inaudito se si ricorda che al monaco non era consentito, dopo il suo ingresso nel monastero, possedere alcunché. E non basta. Alcuni dei girovagi sorrentini avevano approfittato del trambusto dei tempi addirittura per sposarsi. Non si erano accontentati di vivere fuori dalle mura conventuali ma ave-

\footnotetext{
${ }^{24}$ Ibidem, I, 38. 39, a. 591.

${ }^{125}$ Ibidem, IV, 24, a. 594. Sui disordini morali e sugli attacchi alle sanctimoniales, vd. le rigorose disposizioni legislative che comminavano finanche la pena di morte per punire il rapimento e/o lo stupro di una vergine consacrata (Theodosiani libri XVI cum constitutionibus Sirmondinis, in P. Krueger, Th. Mommsen [edd.], Codex Theodosianus, I, Hildescheim, 1990, IX, 25, 1-2, p. 478-479; Codex Justinianus, I, 3, 5, p. 19; IX, 13, p. 378; Novellae, 123, 43, p. 623624; su cui vd. almeno P. LAURENCE, La castitas féminine dans le Code Théodosien: rencontre entre l'Èglise et l'Ėtat?, in J.-N. Guinot, Fr. Richard (éd.), Empire chrétien et Église aux IV et $V^{e}$ siècles. Intégration ou "Concordat»? Le témoignage du Code Théodosien, Actes du Colloque international (Lyon, 6-8 octobre 2005), Paris, 2008, p. 386-387. Per un esame della legislazione conciliare e barbarica in materia, C. URSO, op. cit. (n. 4), p. 75-76, n. 164-166.

${ }^{126}$ GREG. Registrum, V, 19, a. 594.

${ }_{127}$ Ibidem, VIII, 8-9, a. 597. Sull'episodio non si può non citare V. RECCHIA, Monache e monasteri femminili nelle opere di Gregorio Magno. Da un caso di defezione in Puglia nel 597: Ep., VIII, 8; 9 (Hartmann), in Le abbazie nullius. Giurisdizione spirituale e feudale nelle comunità femminili fino a Pio IX, Atti del Convegno di studio (Conversano, 29-31 ottobre 1982), Fasano, 1983, p. 91-130.

${ }^{128}$ GREG. Registrum, IX, 3, a. 598.

${ }^{129}$ Ibidem, IX, 225, a. 599. Nell'episodio sono coinvolti sia Virgilio di Arles sia Siagrio di Autun e ciò non consente di localizzare l'istituto di Siagria in una delle due diocesi: B. JUDIC, L’influence de Grégoire le Grand dans la Provence du VII siècle, in Ch. De Dreuille (éd.), L’Église et la mission au VI siècle. La mission d'Augustin de Cantorbéry et les Églises de Gaule sous l’impulsion de Grégoire le Grand, Actes du Colloque d'Arles de 1998, Paris, 200o, p. 94-95.

${ }^{130}$ GREG. Registrum, X, 3, a. 599.

${ }^{131}$ Ibidem, XI, 26, a. 601.

${ }^{132}$ Ibidem, IX, 108, a. 599: Gregorio denuncia il comportamento scorretto del vescovo di Spoleto, Crisanto.

133 Ibidem, VII, 32, a. 597.

134 Ibidem, V, 33, a. 595.
} 
vano intessuto in maniera indegna pubbliche relazioni con donne del luogo ${ }^{135}$. Aveva preso moglie anche uno dei due monaci fuggiti dal monastero di San Giorgio nella tenuta detta di Marotide in Sicilia, mentre l'altro aveva rinunciato allo status monacale e viveva da laico. Gregorio confutava non solo l'ardire dei due fuggiaschi ma anche le ragioni che costoro, secondo quanto aveva appreso, avanzavano per giustificarsi. Asserivano infatti di essere uomini liberi, non sottoposti a vincoli di tipo servile, non considerando che, se anche così fosse stato, non potevano, dopo essersi liberamente assoggettati al divini officii cultum, tradire il loro Signore ${ }^{136}$.

Molti erano talvolta indotti in tentazione dal contatto con donne che forse non mantenevano un comportamento irreprensibile e consono al luogo: sull'isola quae appellatur Eumorfiana, uomini che possiamo immaginare in fuga dagli attacchi longobardi sulla terraferma si erano sistemati assieme alle loro donne nei pressi di un oratorio. Le probabilità che la quotidiana vicinanza generasse scompiglio fra i monaci lì residenti angustiavano Gregorio che dava mandato al rector del patrimonio campano Antemio di fare cessare lo scandalo, spostando altrove gli indesiderati ${ }^{137}$.

Il malcostume era diffuso anche all'interno dei sacri recinti, come si evince dal corposo elenco dei casi registrati in maniera più o meno dettagliata nell'epistolario gregoriano. Nel 591 l'abate Orosio fu inviato sull'isola di Montecristo, affinché riportasse l'ordine nel monastero isolano ${ }^{138}$; e sempre Orosio fu incaricato di risolvere, con il supporto del defensor romano Simmaco, una situazione analoga sull'isola di Gorgona' ${ }^{39}$. Anni dopo, nel 603, era l'immoralità che regnava tra i monaci che vivevano nell'isola di Cabrera, quae iuxta Maioricam idem insulam, dunque nell'arcipelago delle Baleari, a provocare l'aspra reazione del pontefice ${ }^{140}$.

In un monastero spoletino delle estranee erano state accolte passim (liberamente), i monaci ne avevano scelte alcune come commatres, ovvero come madrine, e «in ragione di ciò si trattengono incautamente con loro»: l'abate Valentino, cui si addossò la responsabilità dell'avvenimento, rischiava, se non fosse stato in grado di porre fine alle incresciose frequentazioni, pene esemplari che fossero da monito agli altri abati $^{141}$. Talvolta la presenza femminile foriera di scandali era correlata alla celebrazione di riti liturgici che attiravano folle di fedeli in servorum Dei recessibus ${ }^{142}$; mentre, a Palermo, erano state le visite sospette del medico Anastasio in un monastero femminile a generare pettegolezzi così molesti da indignare Gregorio e da indurlo a usare parole sferzanti nei confronti di chi, nello specifico il vescovo cittadino Vittore, avrebbe dovuto vegliare sulla fondazione e non se ne era preoccupato ${ }^{143}$.

Di avere commesso un peccato carnale, dum tertius a loco esset abbatis, era reo confesso un tale monaco Secondino, il quale, ciononostante, era stato eletto abate del monastero di San Martino a Napoli ${ }^{144}$. Un notaio che portava lo stesso nome del padre, il vescovo Agnello, aveva stuprato e ingravidato la giovane Petronella, a suo tempo indotta dal prelato ad abbracciare la vita monastica; l'aveva persuasa ad abbandonare il chiostro per scampare ai nemici longobardi e si era portato in Sicilia. In realtà, il notaio aveva anche spinto Petronella a sottrarre al monastero sia i beni personali già devoluti a quest'ultimo, sia quelli che erano stati donati dal vescovo, vale a dire la metà dell'intero suo patrimonio. In tal modo, il notaio riusciva non solo a ricomporre le proprietà paterne, visto che l'altra metà era stata testata proprio a lui, ma anche a disporre di tutti gli averi di Petronella ${ }^{145}$.

A pravis et illecitis actibus si doveva far sì che si astenessero i monaci di Sant'Andrea nell'isola di Vulcano, dove fu chiamato a recarsi il vescovo di Reggio, Giovanni, per verificare realmente la situazione e prendere i necessari provvedimenti ${ }^{146}$. Nel monastero di San Vito sull'Etna, a sentire la segnalazione del monachus Marciano, i religiosi intrattenevano rapporti carnali con donne e il vescovo di Catania, Leone, fino a quel momento - si era nel marzo del 6o4 - non era intervenuto. La circostanza era grave per Gregorio sia se l'ordinario diocesano fosse rimasto inerte perché ignorava i deplorevoli incidenti sia se, invece, pur essendo stato informato, non avesse messo in atto alcuna azione punitiva' ${ }^{147}$.

Altri episodi forse si potrebbero recuperare, ma mi pare più utile riflettere sugli esiti talvolta incredibili dello scadimento morale che compromise in talune occasioni la corretta

\footnotetext{
${ }_{135}$ Ibidem, I, 40, a. 591.

${ }^{136}$ Ibidem, II, 26, a. 592

${ }^{137}$ Ibidem, I, 48, a. 591. Sull'isola vd. supra e n. 48.

${ }^{138}$ Ibidem, I, 49, a. 591.

${ }^{3} 39$ Ibidem, I, 50, a. 591.

${ }^{140} \mathrm{Ibidem}$, XIII, 47, a. 603. Su questo monastero, ubicato in una delle isole Baleari, vd. l'indagine archeologica di M. RIERA RULLAN, El monasterio de la isla de Cabrera (Islas Baleares. Siglos V-VIII d.C.). Testimonios arqueológigos de los monjes reprobados por el papa Gregorio Magno, in Hortus artium medievalium, 19, 2013, p. 47-62, laddove si precisa che la struttura «no pudo ir más allá de las razias efectuadas por los musulmanes en el siglo VIII, o de la definitiva conquista islámica de las Baleares del 902 d.C.» (p. 47).

${ }^{141}$ GREG. Registrum, IV, 40, a. 594.

${ }^{142}$ Ibidem, V, 49, a. 595

143 Ibidem, V, 4, a. 594.

${ }^{144}$ Ibidem, III, 23, a. 593: secondo le indicazioni giunte da Roma, doveva essere Pietro, in quel tempo già rector di Campania, ad allontanare Secondino e a sostituirlo con Teodosio, sicuramente accetto all'intera comunità.

${ }^{145}$ Ibidem, IV, 6, a. 593: Gregorio stabilì che la questione fosse sottoposta al rettore del patrimonio siciliano, Cipriano, perché vagliasse secondo il diritto canonico il comportamento dei due, li punisse con severità e restituisse il maltolto al monastero. La donna, in particolare, doveva essere riportata fra le mura monastiche dopo avere subito la giusta punizione. Sul caso, vd. B. SAITTA, Crisi demografica e ordinamento ecclesiastico nell'Italia di Gregorio Magno, in Romano Barbarica, 19, 2006-2009, p. 205.

${ }^{146}$ GREG. Registrum, V, 55, a. 595.

${ }^{147} \mathrm{Ibidem}$, XIV, 16, a. 604, inviata al vescovo catanese (nella stessa lettera si denunciano tentativi di usurpazione dei beni dello stesso monastero); XIV, 17, a. 604, indirizzata invece al rettore siciliano, notaio Adriano, invitato ad affiancare l'operato di Leone sia nell'impedire attacchi al patrimonio dell'istituto, sia nel correggere i comportamenti dei monaci.
} 
applicazione della normativa regolare. Solo in quest'ottica si spiega l'assurda doppia elezione alla carica di abate avvenuta nello stesso giorno nel monastero palermitano dei Santi Massimo e Agata, o Lucuscano. L'abate Urbico, peraltro nel contempo accusato di trattenere indebitamente 40 solidi spettanti al suddetto monastero, era stato chiamato a presiedere alla scelta e aveva indicato come candidato il presbitero Domizio al mattino, e alla sera il monaco Bono. Nella lettera si denuncia non a caso la disobbedienza dei monaci e, di conseguenza, l'incapacità di Urbico di imporre le sue decisioni, causando così un danno all'istituzione ${ }^{148}$. Qualcosa di simile accadde anche nel monastero femminile di San Martino dove, secondo la nostra ricostruzione, una monaca, di nome Vittoria, organizzò impunemente il trasferimento coatto presso un'altra comunità della "collega" Marcia. Costei locum illius sequebatur ed era perciò una pericolosa concorrente di Vittoria nella sua corsa verso il titolo di priora, una carica molto ambita perché si collocava al gradino immediatamente inferiore a quello di badessa. Il suo piano, peraltro, era stato perfezionato con la distribuzione di beni dell'istituto per accattivarsi il sostegno di personaggi la cui identità bisognava fosse scoperta dal defensor Fantino, mentre, in attesa dei risultati dell'indagine, Vittoria era inviata presso un altro monastero ${ }^{149}$.

Quest'ultimo dettaglio ci è utile per ricordare l'utilizzo delle case monastiche più rinomate per la loro integrità morale come luoghi di segregazione e di penitenza, laddove i colpevoli, laici ed ecclesiastici, di gravi reati avevano l'opportunità di riconsiderare la loro condotta. Ospiti "speciali" di queste rinomate strutture - ad esempio il monastero di Monosteo in Sicilia o quello di San Giovanni in Classe a Ravenna -, alle quali per inciso erano rimesse le dotazioni personali di tali reclusi oppure precise sovvenzioni de reditibus sanctae Romanae ecclesiae perché potessero affrontare le spese dell'accoglienza, furono monache e monaci, così come vescovi (anche il vescovo di Zara Sabiniano fu così punito nel 597 per avere avuto rapporti con Massimo, usurpatore della chiesa di Solona), e laici, colpevoli di stupro o di altri gravi reati ${ }^{150}$.

\section{CONCLUSIONI}

Nonostante tutto, dunque, Gregorio continuava a nutrire fiducia nel monachesimo e nei confronti di chi abbracciava l'ideale monastico eai suoi contenuti improntava il suo comportamento e i suoi pensieri. Su costoro puntò per attuare taluni progetti prettamente pastorali, che rientravano cioè nella politica generale del pontificato e non nella politica monastica vera e propria, e per questo sono qui ripresi solo per rimarcare il rapporto stretto fra Gregorio e gli ambienti monastici e le speranze che egli riponeva negli esponenti del movimento più noti per santità di vita e per capacità organizzativa. Il riferimento è ovviamente all'impresa del monaco Agostino e dei suoi compagni presso gli Angli ealle missioni svolte da alcuni monaci presso la corte longobarda ${ }^{151}$.

Nonostante tutto i monasteri, quelli di risaputa irreprensibilità e santità di vita, erano per lui i luoghi più sicuri per ritrovare la giusta via e continuare a seguirla. Era indispensabile perciò impedire che la dignitas monacale fosse incrinata. Quando ciò si verificava urgevano provvedimenti immediati e fermi. In Isauria il monaco Atanasio, insignito anche dell'ordinazione sacerdotale, era stato fustigato nel silenzio totale del patriarca di Costantinopoli Giovanni, sotto la cui giurisdizione ricadeva la chiesa locale. Gregorio respinse prontamente le ragioni della latitanza del patriarca nella vicenda, scaturite, secondo la missiva recapita a Roma, dalla mancanza di informazioni e, pur dicendosi convinto che a scrivere non fosse stato il suo esimio interlocutore bensì un giovane, sprovveduto ma superbo segretario, non lesinò a Giovanni i suoi aspri e taglienti commenti: «A questa risposta rimasi meravigliato moltissimo, pensando tra me: se dice il vero, che cosa può esserci di peggio che

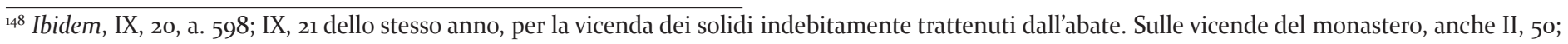
VI, 49; IX, 67, per un arco di tempo che va dal 592 al 598.

${ }^{149}$ Ibidem, V, 4, a. 594.

${ }^{150}$ Ibidem, I, 42, a. 591: sulla monaca che, caduta in peccato, era stata trasferita nel monastero di Monosteo in Sicilia; V, 25, a. 595: sul monaco Valeriano che scontava la sua penitenza nel monastero ravennate di San Giovanni in Classe. E ancora: il vescovo girovagus che Gregorio ordina, in caso di recidiva, sia rinchiuso in un monastero è quello di Amalfi, Pimenio (VI, 23, a. 596); scontò in un monastero la penitenza inflittagli per avere avuto pericolosi legami con Massimo usurpatore della Chiesa di Solona, il vescovo di Zara Sabiniano (VIII, 11, a. 597); ad eguale destino erano avviati i presbiteri e i diaconi del vescovo di Malta Lucillo, accusati di gravi reati commessi in concorso col vescovo e con alcuni laici (IX, 25, a. 598). A proposito di laici condannati a scontare la pena in un monastero, un tale Felice fu minacciato di essere internato, se si fosse rifiutato di sposare, dopo averla violentata, la figlia del diacono di Siponto, Evangelo (III, 40, a. 593; lo stupro era condannato dal diritto romano anche con la pena capitale: supra n. 125); Onorata, vedova del suddiacono Specioso, fu confinata ingiustamente, vista la normativa allora vigente in Sicilia, dal vescovo catanese Leone in una struttura monastica, per essersi risposata dopo la morte del marito (IV, 34, a. 594); e, per altre simili vicende: V, 17-19, a. 594; e supra, n. 127 sulla vicissitudine della figlia di Tulliano. Sul tema nelle sue più ampie articolazioni, vd. specialmente K. L. NOETHLICHS, Das Kloster als 'Strafanstalt' im kirchlichen und weltlichen Recht der Spätantike, in Zeitschrift der Savigny-Stiftung für Rechtgeschichte, Kan. Abteilung, 80, 1994, p. 18-40; J. HILLNER, Gregory the Great's "prisons": monastic confinement in early byzantine Italy, in Journal of Early Christian Studies, 19, 3, 2011, p. 433-471, dove si sottolinea che «The function that Gregory assigned to monastic confinement was certainly religieuse. Monastic confinement had a very firm place in his program of pastoral flexibility» (p. 437).

${ }^{151}$ Sul tema, che qui non sarà affrontato, segnalo (oltre al recente saggio di C. RICCI, op. cit. [n. 13, p. 47-55, laddove si ribadisce che Gregorio nelle sue iniziative di proselitismo non perseguì un programma ideologico bensì pastorale) la riflessione di G. BARONE (Cristianesimo e identità europea, in Storia d'Europa e del Mediterraneo, II. A. Barbero [dir.], Dal Medioevo all'età della globalizzazione, sez. IV: S. Carocci [a cura di], Il Medioevo [secoli V-XV], VIII: Popoli, poteri, dinamiche, Roma, 2006, p. 8o-83) per la quale Gregorio si impegnò nella conversione degli Angli, trascurando invece quella dei Longobardi perché questi ultimi «erano ariani, perciò non ortodossi, ma comunque cristiani. La salvezza era stata loro assicurata dal battesimo, contrariamente a quanto avveniva per i pagani». Ė un approccio forse incompleto - non tiene conto ad esempio delle accuse di idolatria rivolte dal papa al popolo longobardo, che dunque era ancora considerato per certi versi "pagano" (vd. in tal senso, N. EVERETT, Literacy in Lombard Italy, c. 568-774, Cambridge, 2003, p. 59-62) - ma certo originale alla vexata quaestio. Sui rapporti fra Gregorio, Teodolinda e l'abate Secondo: GREG. Registrum, IX, 148, a. 599; XIV, 12, a. 603; W. POHL, Gregorio Magno e il regno dei Longobardi, in C. Azzara (a cura di), Gregorio Magno, l'impero e $i$ «regna», Atti dell'incontro internazionale di studio dell'Università degli studi di Salerno - Osservatorio dell'Appennino Meridionale, con la collaborazione della Fondazione Ezio Franceschini e della SISMEL (Fisciano, 30 settembre-1 ottobre 2004), Firenze, 2008, p. 26, n. 47; C. URSO, op. cit. (n. 4), p. 193-194, n. 46o-461. 
si compiano tali cose contro dei monaci e non ne sappia niente chi è presente? Quale, infatti, può essere la scusa del pastore, se il lupo si mangia le pecore e il pastore non lo sa? Se poi la santità tua sapeva, e il motivo per cui le scrissi e ciò che fu fatto [...] e mi scrisse: non lo so. Che cosa risponderò contro questo fatto, dal momento che la Verità per mezzo della Scrittura sua dice: La bocca che mente uccide l'anima? $\gg^{152}$.

Era angosciato Gregorio, i problemi erano tanti e talvolta apparivano insormontabili; egli si muoveva inseguendo precisi obiettivi ma gli uomini che avrebbero dovuto sostenere le sue azioni si dimostravano troppo spesso impreparati o, peggio, conniventi. «Impegnato sino a una dedizione eroica nella cura animarum, Gregorio Magno avvertiva con sofferenza il peso della pastorale sollecitudine verso la Chiesa e il mondo [...] egli si sente - indignus infirmusque - alla guida di una compagine paragonata a una nave colpita dalla tempesta, squarciata, vetusta e aggredita dai flutti, come nell'imminenza di un naufragio» ${ }^{153}$.

E il mondo monastico non era certo paragonabile ad un'isola felice. Viveva anzi una «fase di stanca»"154; e tuttavia, e mi piace concludere con queste considerazioni cui giungevo qualche anno fa, «tutte le criticità riscontrate [...] sono da considerarsi indizi di un processo inesorabile di declino o non piuttosto l'emergere di fenomeni fisiologici, se pur gravi, connaturati con la pienezza di un movimento che, avendo cominciato ad assumere caratteri quasi di massa, non poteva non sperimentare a latere siffatte crisi di ordine morale e socio-economico, senza doverne per forza essere travolto o adulterato» ${ }^{155}$ ?

\footnotetext{
${ }^{152}$ GREG. Registrum, III, 52, a. 593; sulla questione dei monaci dell'Isauria tornano l'ep. V, 44, a. 595, dalla quale si evince che il patriarca costantinopolitano aveva in qualche modo chiarito la sua posizione, e l'ep. VII, 31, a. 597, indirizzata in copia uniforme ai patriarchi di Alessandria e di Antiochia.

${ }^{153}$ G. CREMASCOLI, op. cit. (n. 6), p. 661, dove si commenta così il contenuto della lettera a Teoctista, sorella dell'imperatore Maurizio (GREG. Registrum, I, 5, a. 591).

154 R. TURTAS, Note sul monachesimo in Sardegna, op. cit. (n. 37), p. 109.

${ }^{155}$ C. URSO, op. cit. (n. 4), p. 92.
}

\begin{tabular}{|l|c|c|c|c|}
\hline Hortus Artium Mediev. & Vol. 23/1 & $31-49$ & C. Urso & STRUTTURE MONASTICHE... \\
\hline
\end{tabular}

